\title{
An atlas of images of Planetary Nebulae ${ }^{\star}$
}

\author{
S.K. Górny ${ }^{1}$, H.E. Schwarz ${ }^{2}$, R.L.M. Corradi ${ }^{3}$, and H. Van Winckel ${ }^{4}$ \\ 1 Copernicus Astronomical Center, Laboratory of Astrophysics, ul. Rabiańska 8, PL-87-100 Toruń, Poland \\ e-mail: skg@ncac.torun.pl \\ 2 Nordic Optical Telescope, Apartado 474, E-38700 Sta. Cruz de La Palma, Spain \\ 3 Instituto de Astrofisica de Canarias, 38200 La Laguna, Tenerife, Spain \\ ${ }^{4}$ KU Leuven, Celestijnenlaan 200B, Leuven, Belgium
}

Received November 2; accepted December 23, 1998

\begin{abstract}
A catalogue of narrowband CCD images of 100 southern and 1 northern Planetary Nebulae is presented. The data are images taken in the light of the $\mathrm{H} \alpha, \mathrm{H} \beta$, [OIII] $500.7 \mathrm{~nm}$, and [NII] $658.4 \mathrm{~nm}$ lines. For each image we give the exposure time, the maximum extent of the nebula, and the seeing measured from the frames themselves. For ease of reference, we have added an alphabetical list of the objects. Our list will be useful for morphological studies of Planetary Nebulae, and is complementary to previously published lists, especially the one of Schwarz et al. (1992).
\end{abstract}

Key words: atlases — planetary nebulae

\section{Introduction}

Planetary nebulae $(\mathrm{PNe})$ are of importance to astrophysics for various reasons: they form the spectacular evolutionary end point for most low to intermediate mass stars, they inject a dominant fraction of the enriched elements into the interstellar medium, and they allow us to study many physical problems of plasma dynamics, kinematics, emission line formation including forbidden lines etc.

Recently, the study of the morphology of these nebulae has come of age. Links are being made between the appearance of PNe and their central star's position in the HR diagram, in other words between their evolutionary stage and their shape (Stanghellini et al. 1995); the properties of bipolar nebulae have been shown to be rather special and related to various other physical parameters of the objects (Corradi \& Schwarz 1995), and

Send offprint requests to: S.K. Górny

* Based on observations obtained at the European Southern Observatory, La Silla, Chile.
Table 1. Telescopes, CCDs and pixel scales used

\begin{tabular}{clll}
\hline code & telescope & instrument & CCD pixel scale (arcsec) \\
\hline a & Dutch $0.91 \mathrm{~m}$ & Adapter & GEC 0.'36 \\
b & Danish $1.54 \mathrm{~m}$ & Adapter & TEK $0^{\prime \prime} 43$ \\
$\mathrm{c}$ & ESO 3.6 m & EFOSC & RCA $0^{\prime \prime} 61$ \\
\hline
\end{tabular}

dedicated conferences are being held on the subject of PNe morphology, especially asymmetries (see e.g. Harpaz \& Soker 1994). Clearly, it is of importance for any studies like the above mentioned ones to have access to a sample of narrow band images that is statistically as complete as possible.

Previous well-known catalogues of narrowband images have been published by Balick (1987), Schwarz et al. (1992), and Manchado et al. (1996) and there are many others referenced therein. All these catalogues take their objects from the large catalogue of Acker et al. (1992). Here we add some 100 southern and 1 northern PNe to the data base.

\section{Observations}

The observations have been made at the European Southern Observatory with several different telescopes and instruments, and over a period of a few years. In all cases the detector was a CCD, and the great majority of the images has been secured with the Dutch $91 \mathrm{~cm}$ telescope. In Table 1 we list the instrumental parameters for all the observations.

The observations have all been made during the 1993 to 1995 period. Data have been taken through narrow band filters centred on the following emission lines: $\mathrm{H} \alpha 656.3 \mathrm{~nm}, \mathrm{H} \beta 486.1 \mathrm{~nm},[\mathrm{OIII}] 500.7 \mathrm{~nm}$, and [NII] $658.4 \mathrm{~nm}$. The FWHM of the filters were typically $3 \mathrm{~nm}$, so some contamination of the [NII] images with $\mathrm{H} \alpha$ 
light is present but at low level (about 5\%). Nearly all objects have an $\mathrm{H} \alpha$ image but only some have had the other lines recorded. In total we present 198 images of 100 southern and 1 northern PN.

All data were bias subtracted and flat fielded in the usual way but no flux calibration was done.

\section{Results}

The results are presented in Figs. 1 to 33. A few comments where appropriate have been given below. Note that each image has a size bar superimposed on it, to make scaling of the objects easy. The size of the bars varies but in all cases the projected length is $20^{\prime \prime}$. For many objects with a wide dynamical range of emission we plot their images with a double grey scale to show better the details of the bright region. In all cases north is up, and east is to the left.

In Table 2 we list the observed objects. We give: index number, RA and Dec (2000.0), Galactic coordinate name, corresponding Figure No., image sizes and seeing FWHM on the same frame for all filters taken.

In Table 3 we give an alphabetical list of object names to make searching for a given $\mathrm{PN}$ easier.

Comments on Table 2:
1. The $\mathrm{H} \beta$ image probably has been taken through an $\mathrm{H} \alpha$ filter by mistake, hence the strong similarity between the two images presented.

2. Possible planetary nebulae according to Acker et al. (1992).

Acknowledgements. S.K. Górny acknowledges the Nordic Optical Telescope Scientific Association for providing the opportunity to work on these data at La Palma and financial support from the grant No. 2.P03D.027.10 of the Polish Committee for Scientific Research.

\section{References}

Acker A., Ochsenbein F., Stenholm B., Tylenda R., Marcout J., Schon C., 1992, Strasbourg-ESO Catalogue of Galactic Planetary Nebulae, European Southern Observatory

Balick B., 1987, AJ 94, 671

Corradi R.L.M., Schwarz H.E., 1995, A\&A 293, 871

Harpaz A., Soker N., 1994, Ann. Isr. Phys. Soc. 11, Asymmetrical Planetary Nebulae, Oranim, University of Haifa

Manchado A., Guerrero M.A., Stanghellini L., Serra-Ricart M., 1996, The IAC Morphological Catalogue of Northern Galactic Planetary Nebulae, Instituto de Astrofísica de Canarias

Schwarz H.E., Corradi R.L.M., Melnick J., 1992, A\&AS 96, 23

Stanghellini L., Corradi R.L.M., Schwarz H.E., 1993, A\&AS 279,521 

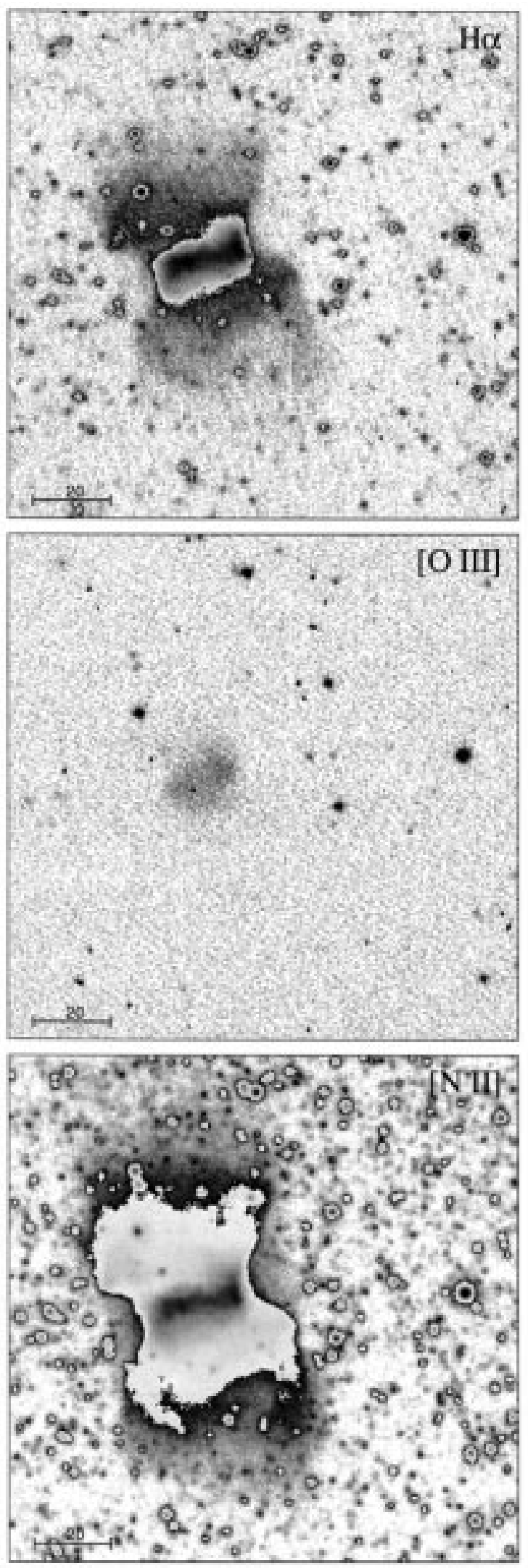
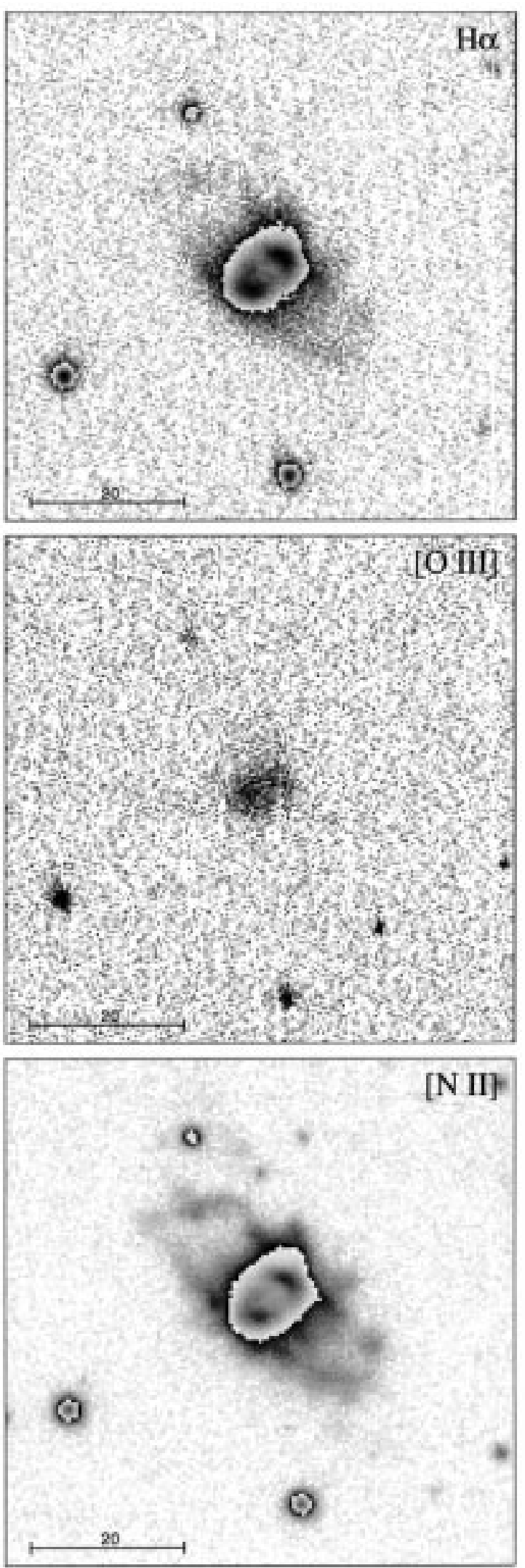

Fig. 1. M 1-28 (left), and M 3-2 (right) 

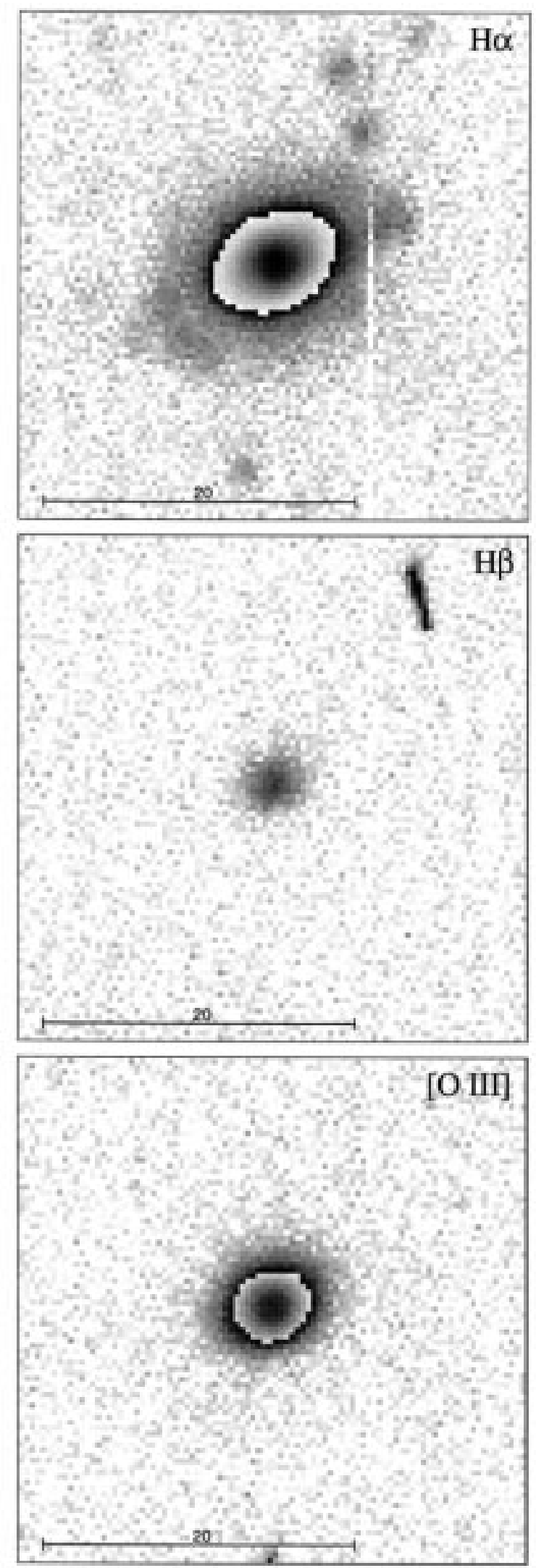

Fig. 2. He 2-67 (left), and NGC 3195 (right)
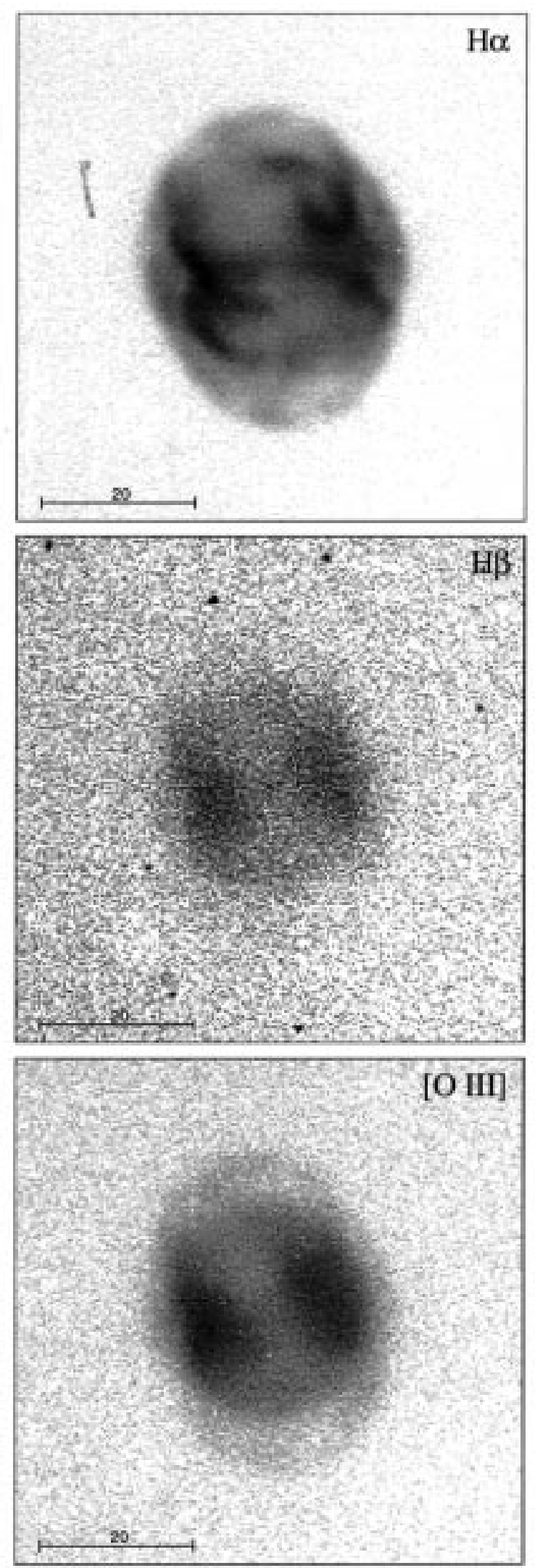

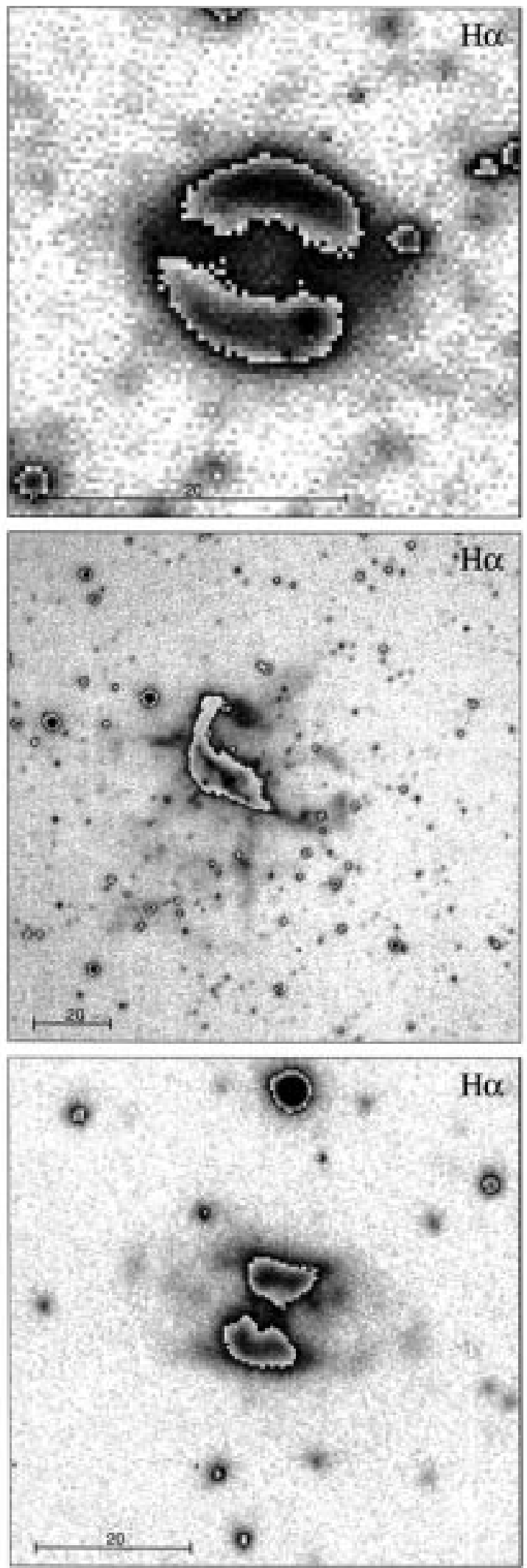

Fig. 3. H 1-51 (top), He 2-70 (middle), and He 2-76 (bottom)
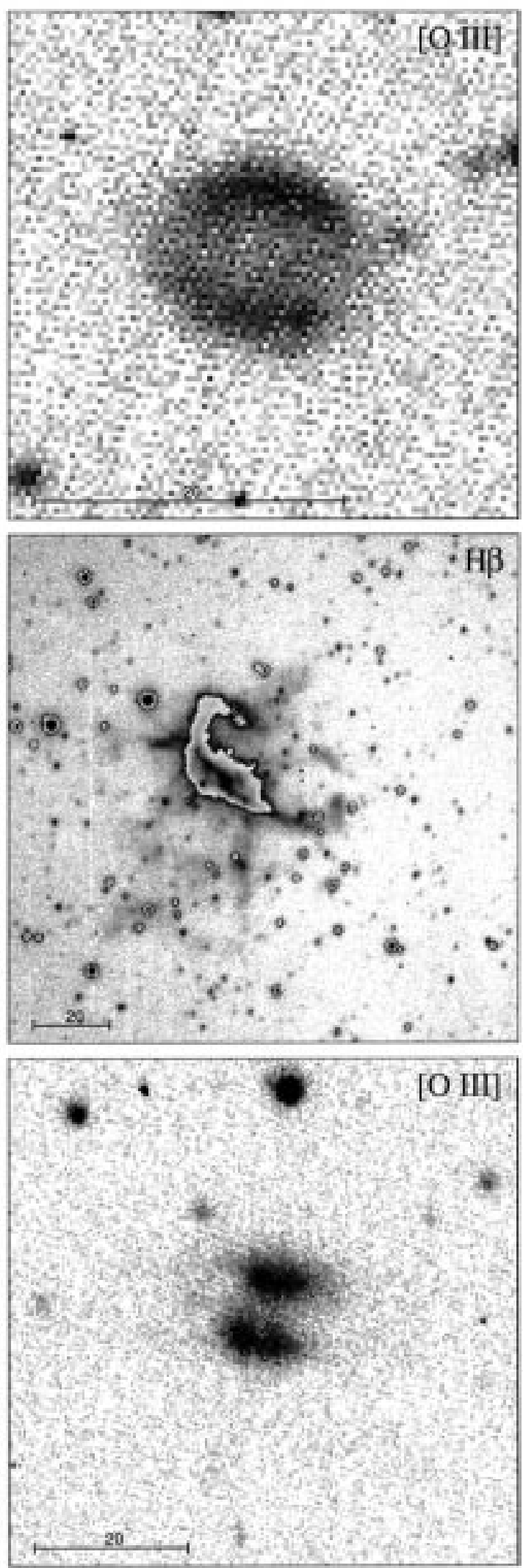

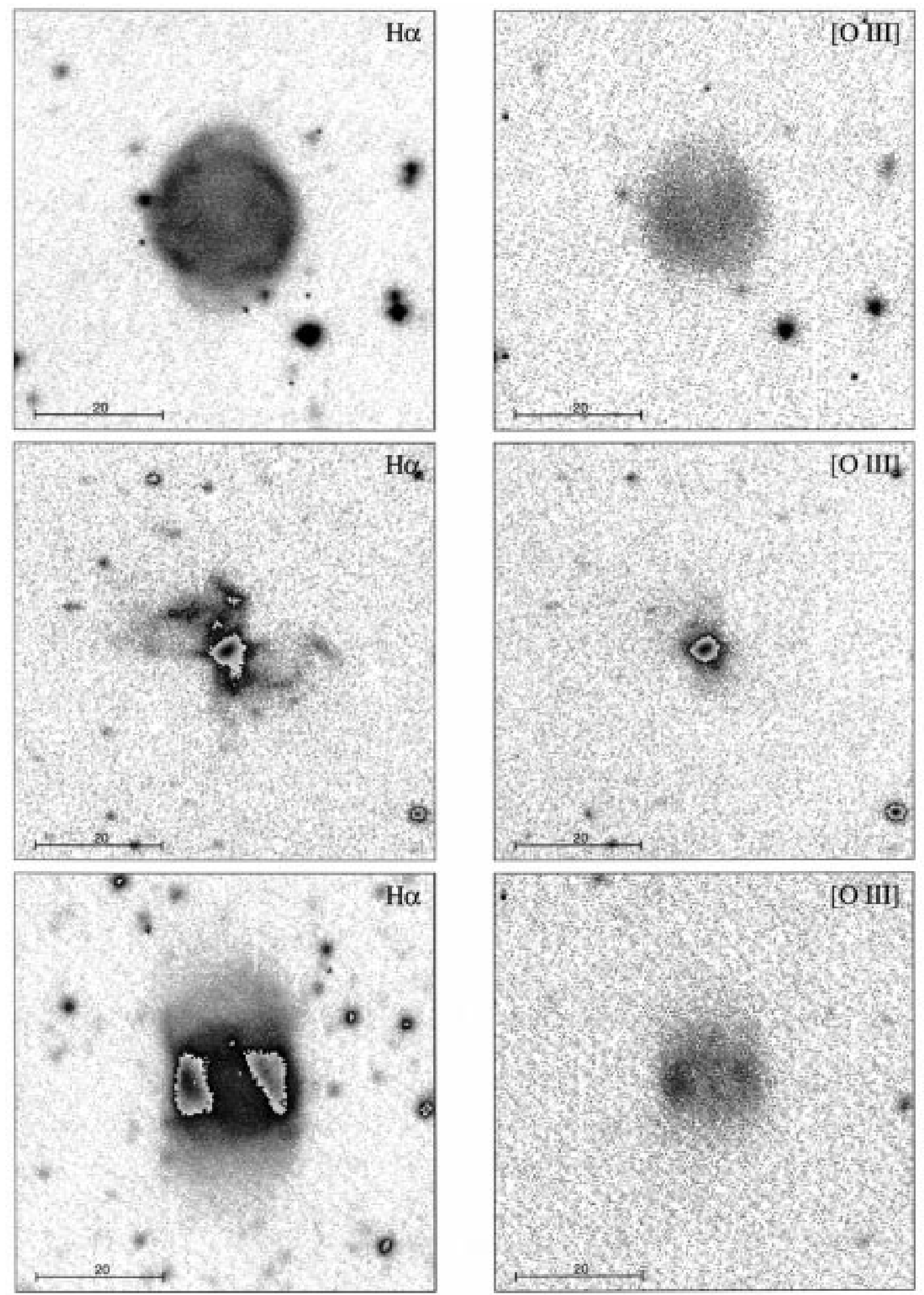

Fig. 4. He 2-82 (top), He 2-84 (middle), and He 2-114 (bottom) 

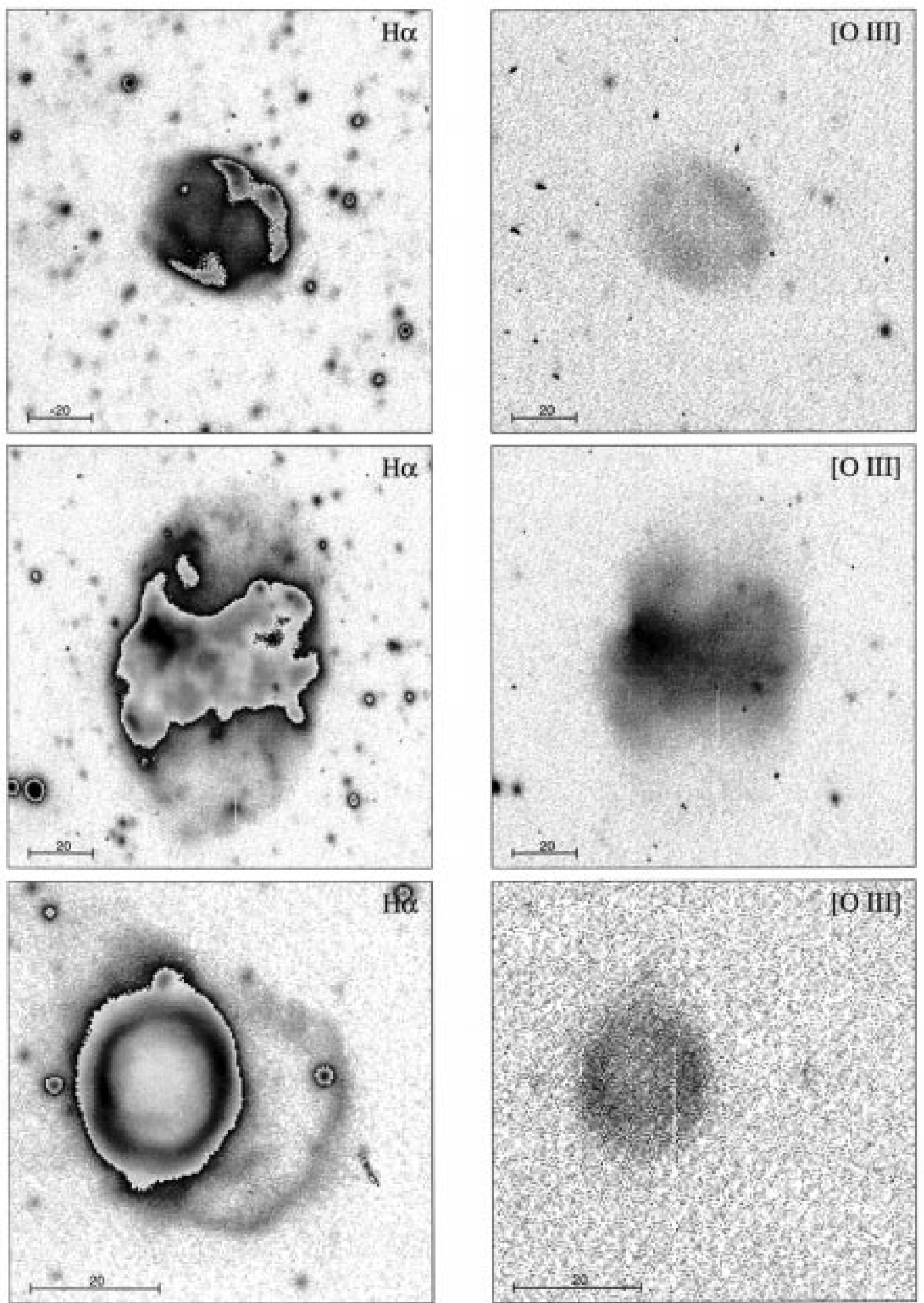

Fig. 5. He 2-116 (top), He 2-119 (middle), and He 2-120 (bottom) 

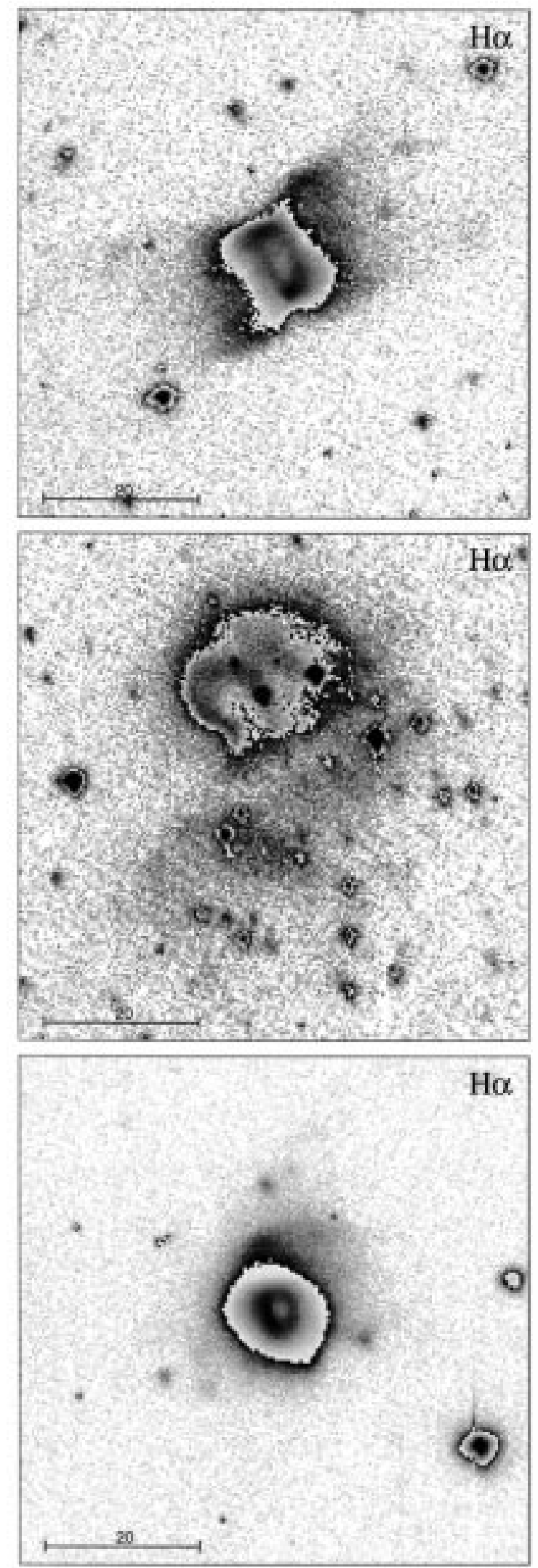
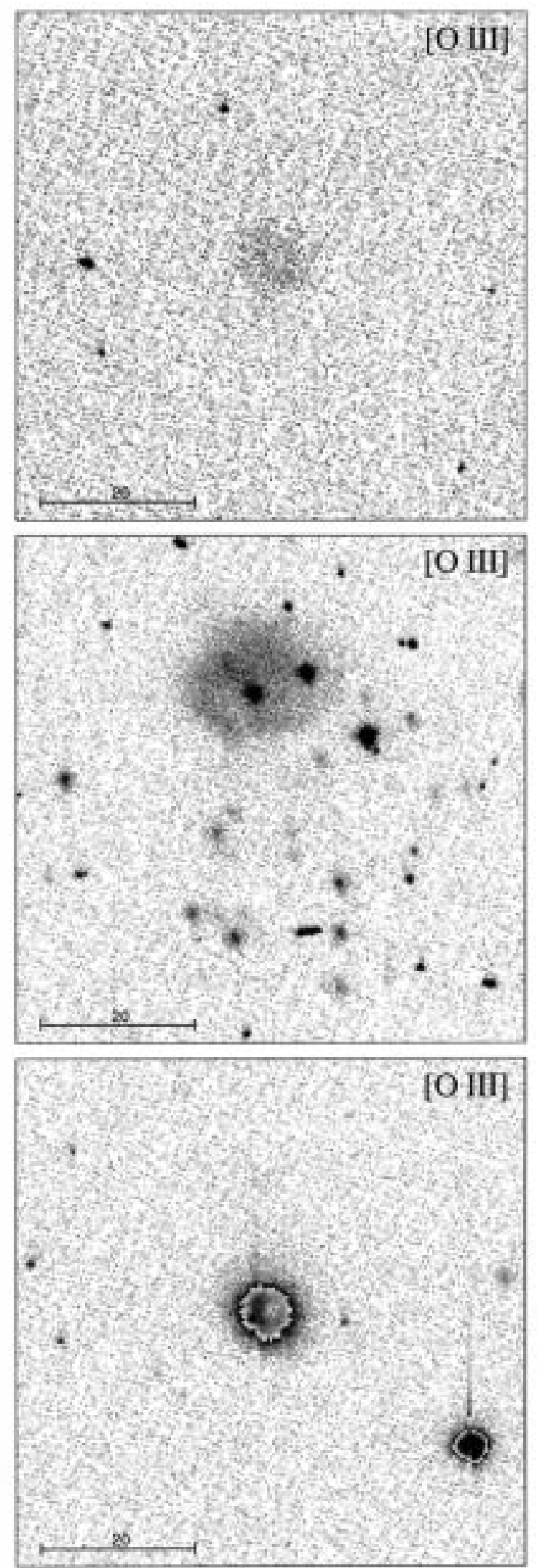

Fig. 6. He 2-145 (top), He 2-146 (middle), and He 2-152 (bottom) 

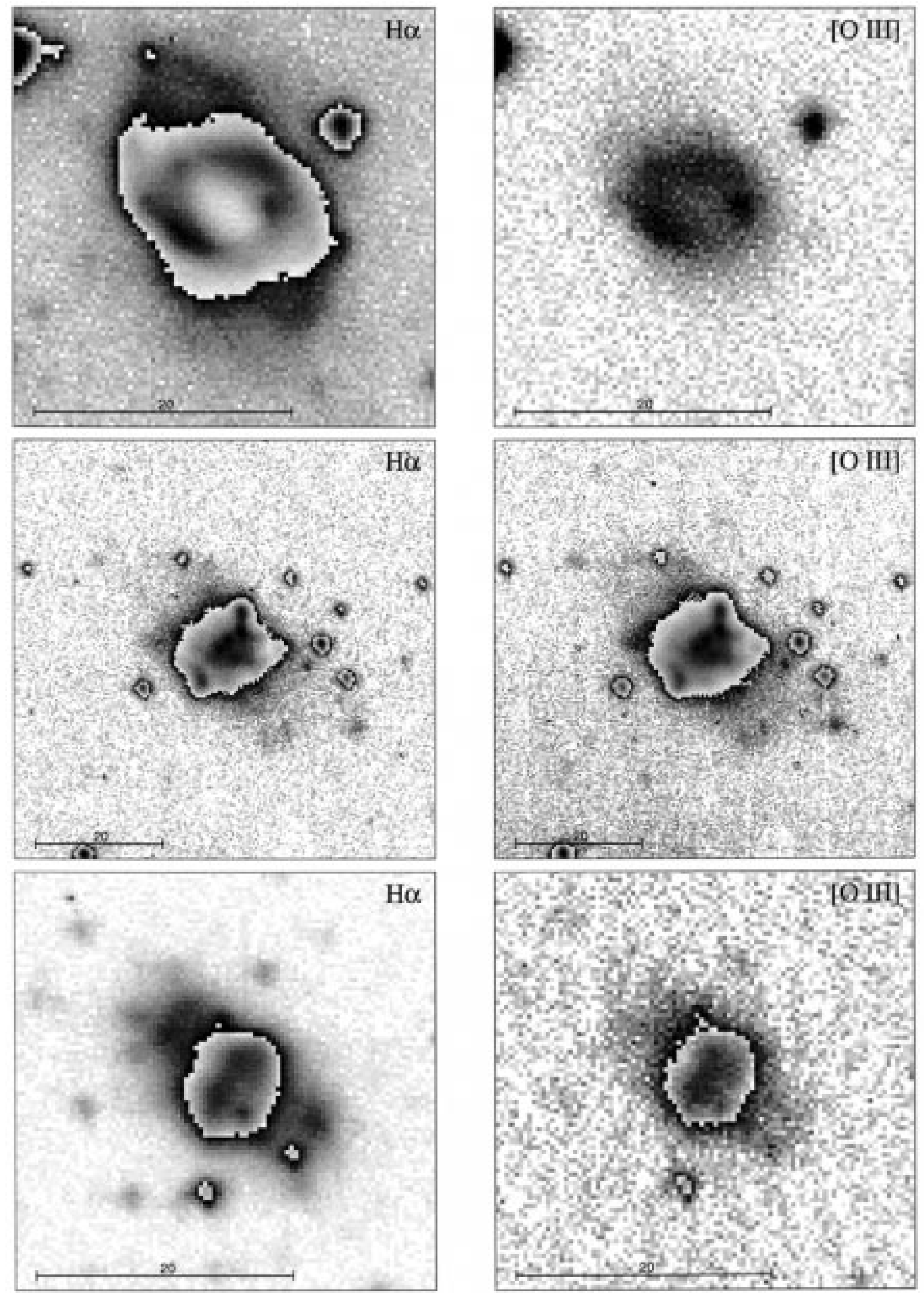

Fig. 7. He 2-153 (top), He 2-155 (middle), and He 2-161 (bottom) 

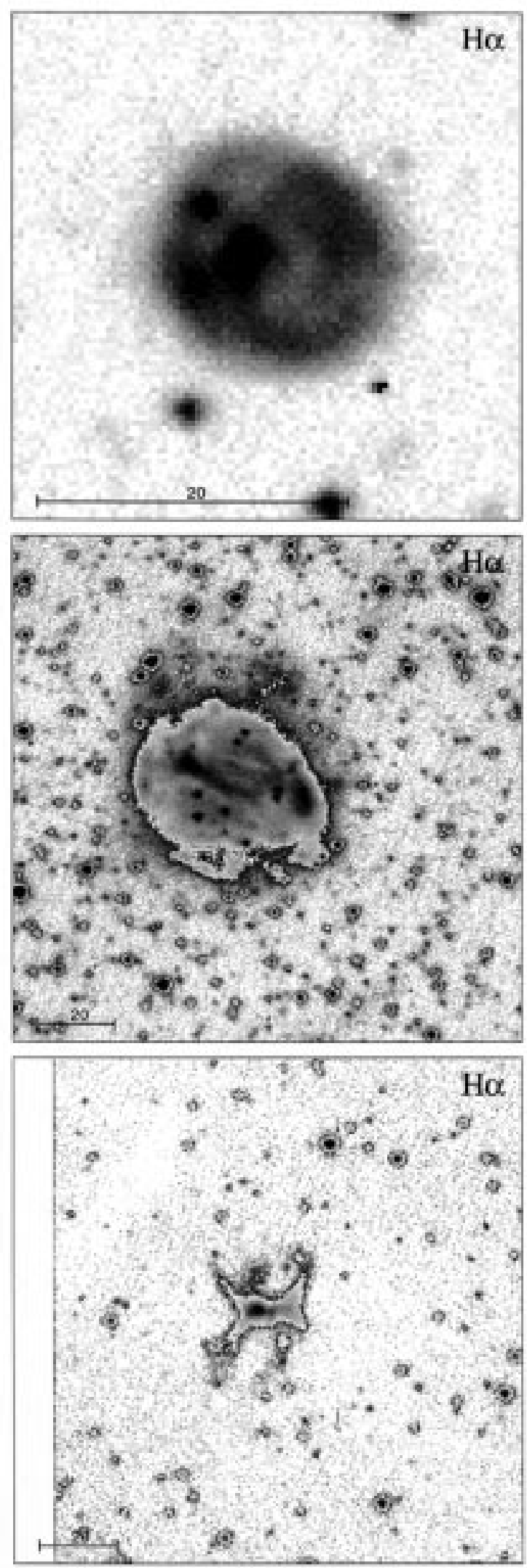
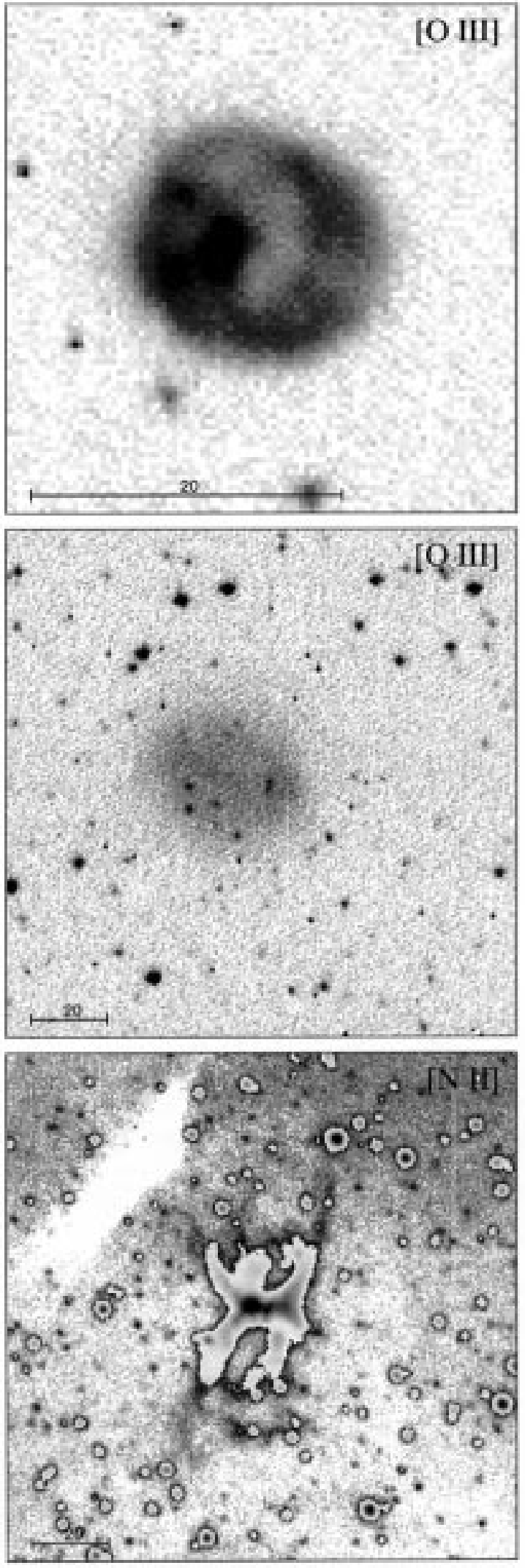

Fig. 8. He 2-164 (top), He 2-165 (middle), and He 2-169 (bottom) 

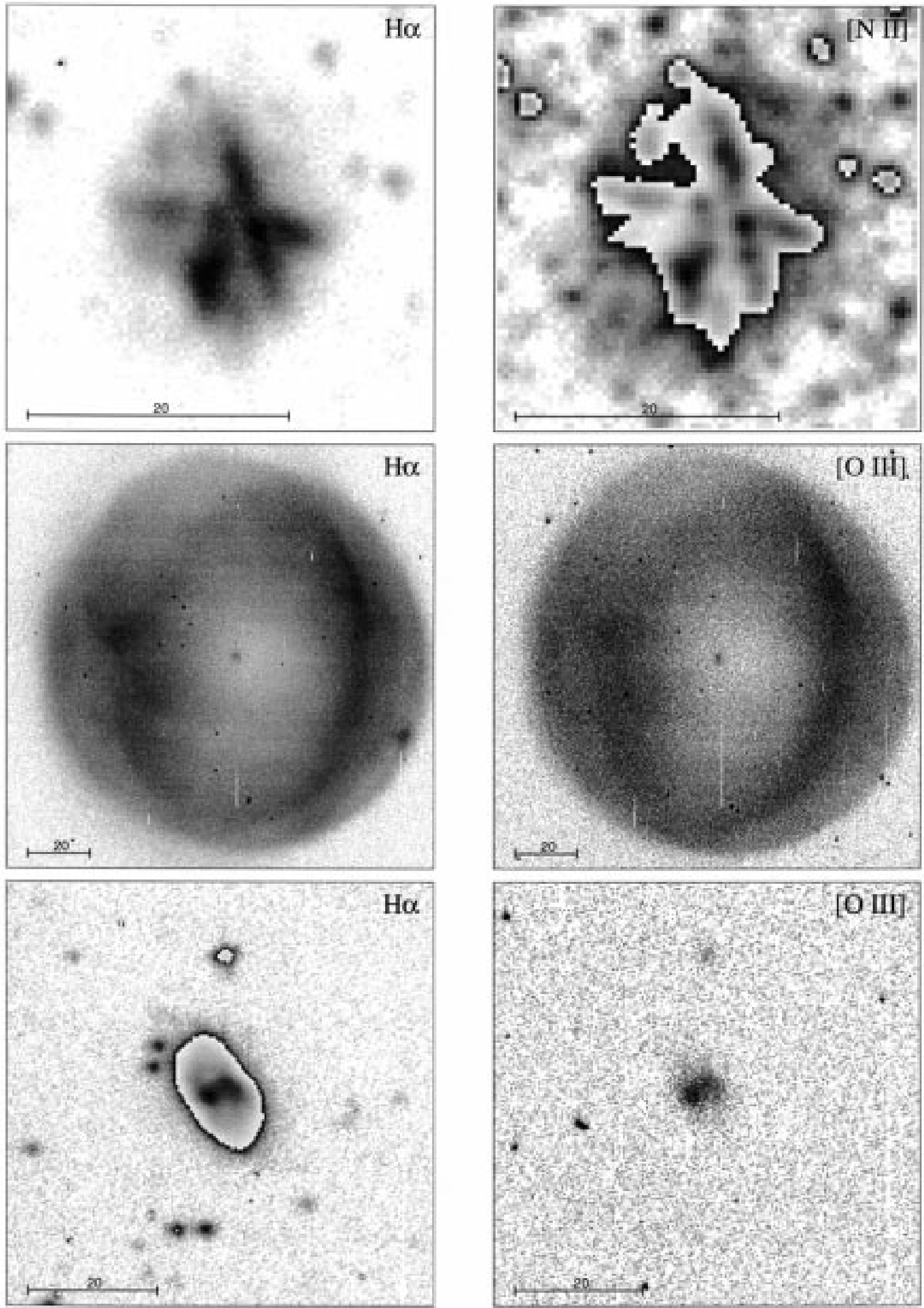

Fig. 9. Hf 2-1 (top), Ic 4148-50 (middle), and M 1-151 (bottom) 

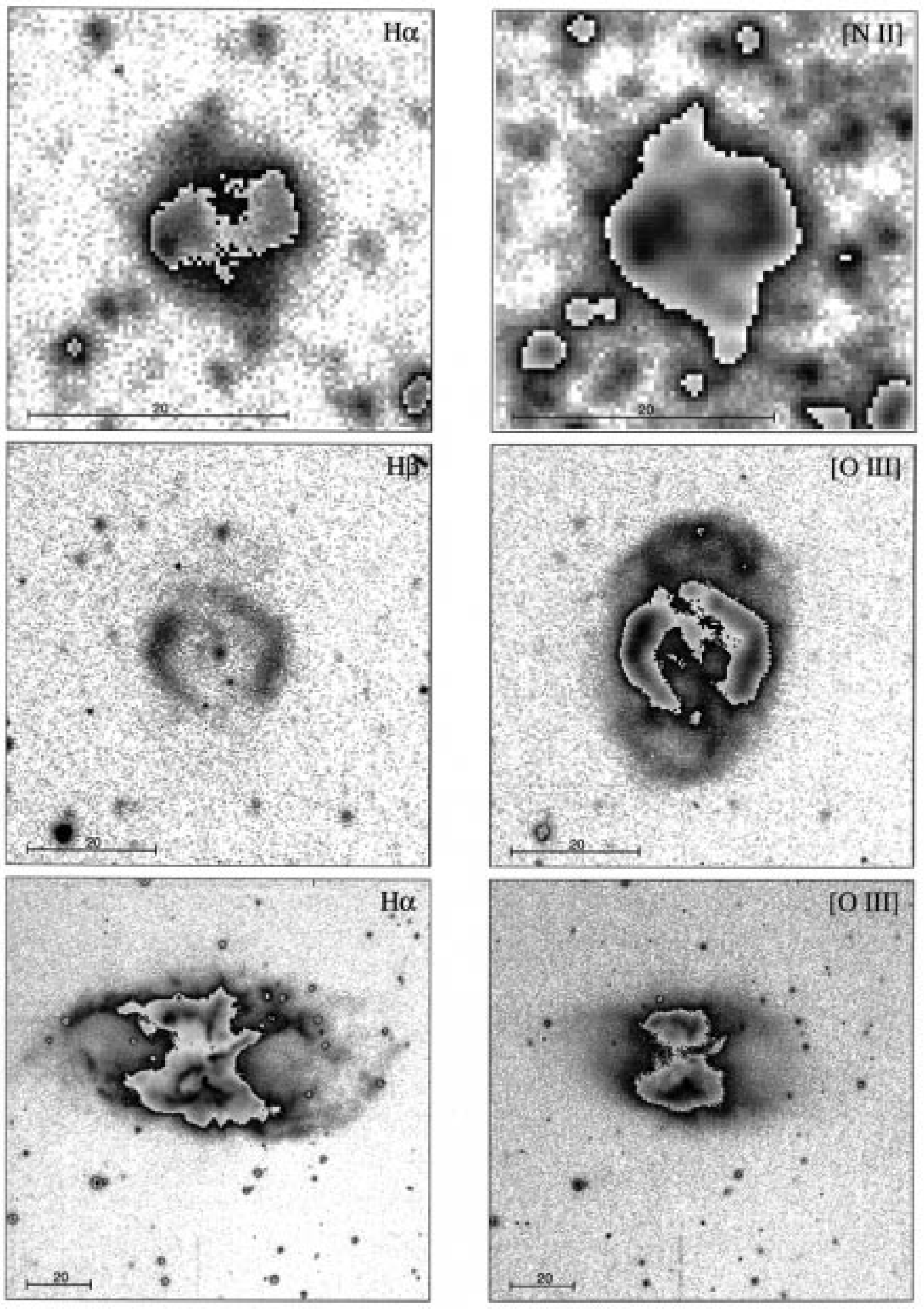

Fig. 10. M 3-51 (top), Mz 2 (middle), and NGC 2818 (bottom) 

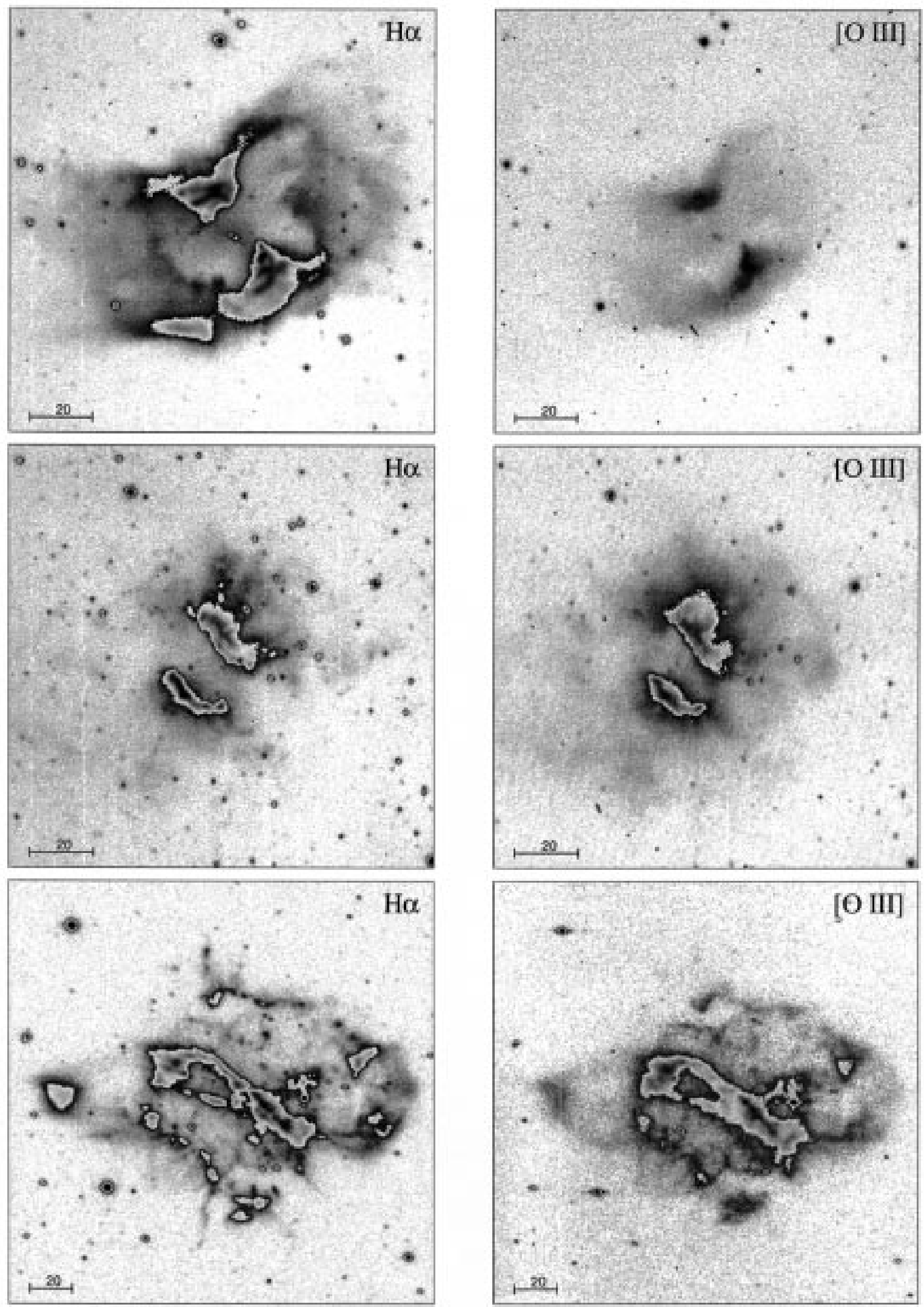

Fig. 11. NGC 2899 (top), NGC 3699 (middle), and NGC 5189 (bottom) 

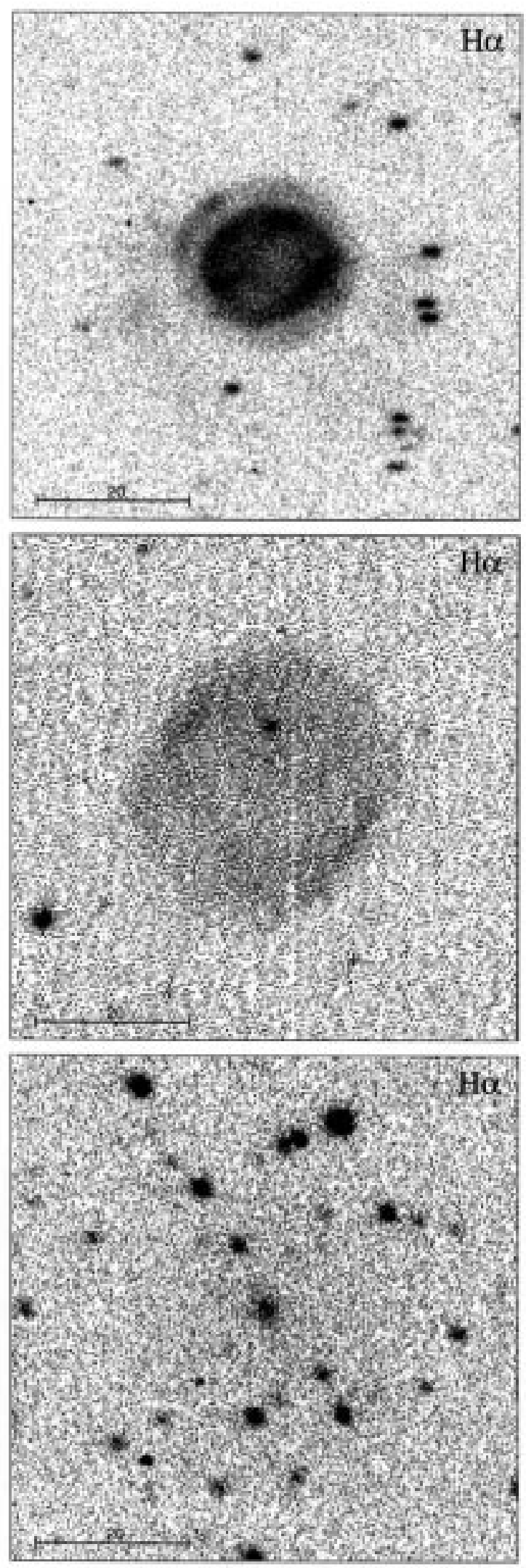
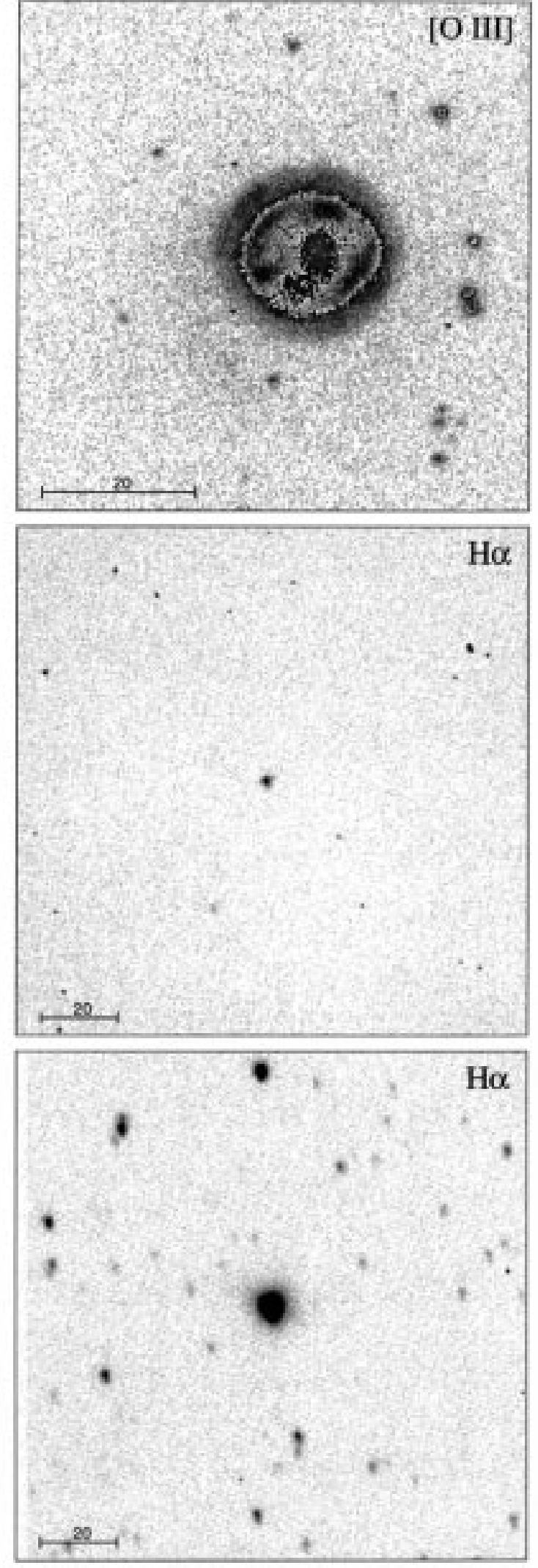

Fig. 12. Pe 1-8 (top), A 26 (middle left), A 7 (middle right), Anon. 19h19m (bottom left), Cn 1-1 (bottom right) 

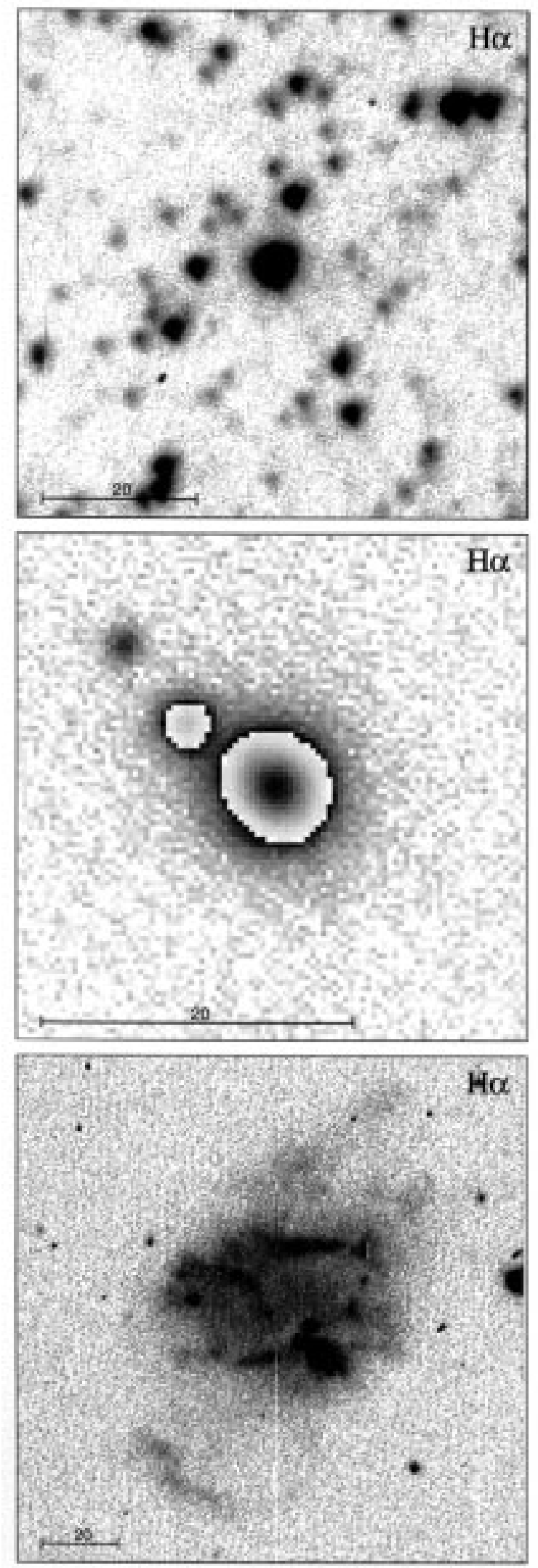

Fig. 13. H 1-43 (top), Hb 7 (middle), and He 2-11 (bottom)
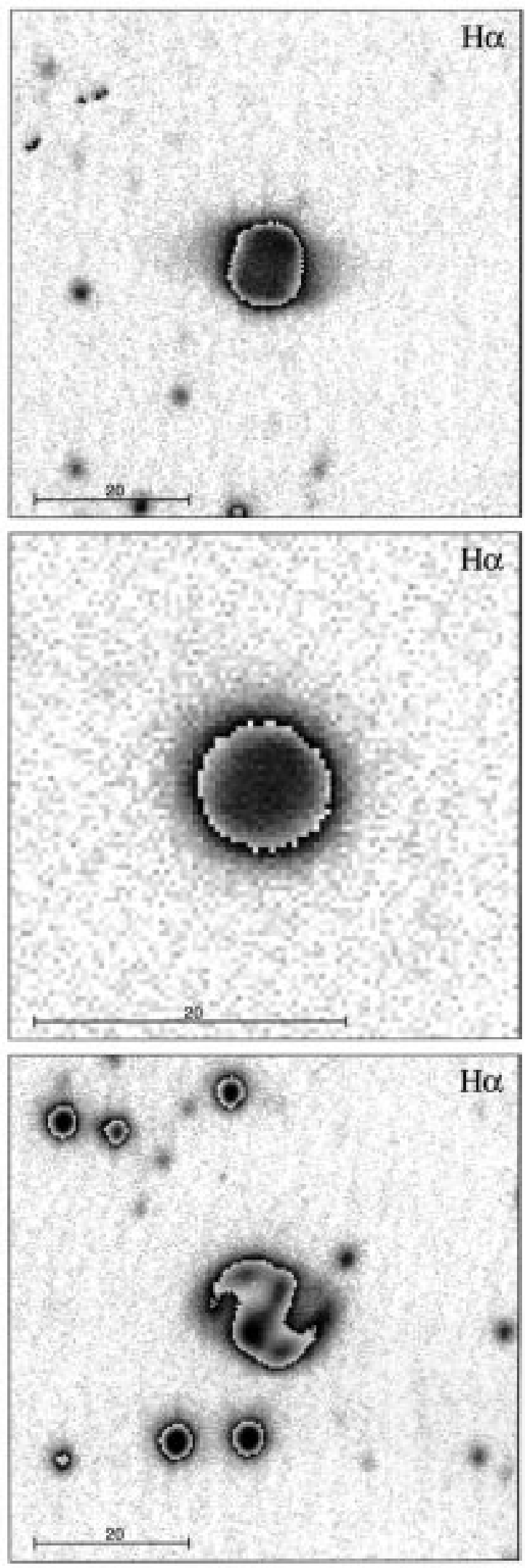

Fig. 14. He 2-18 (top), He 2-28 (middle), and He 2-29 (bottom) 

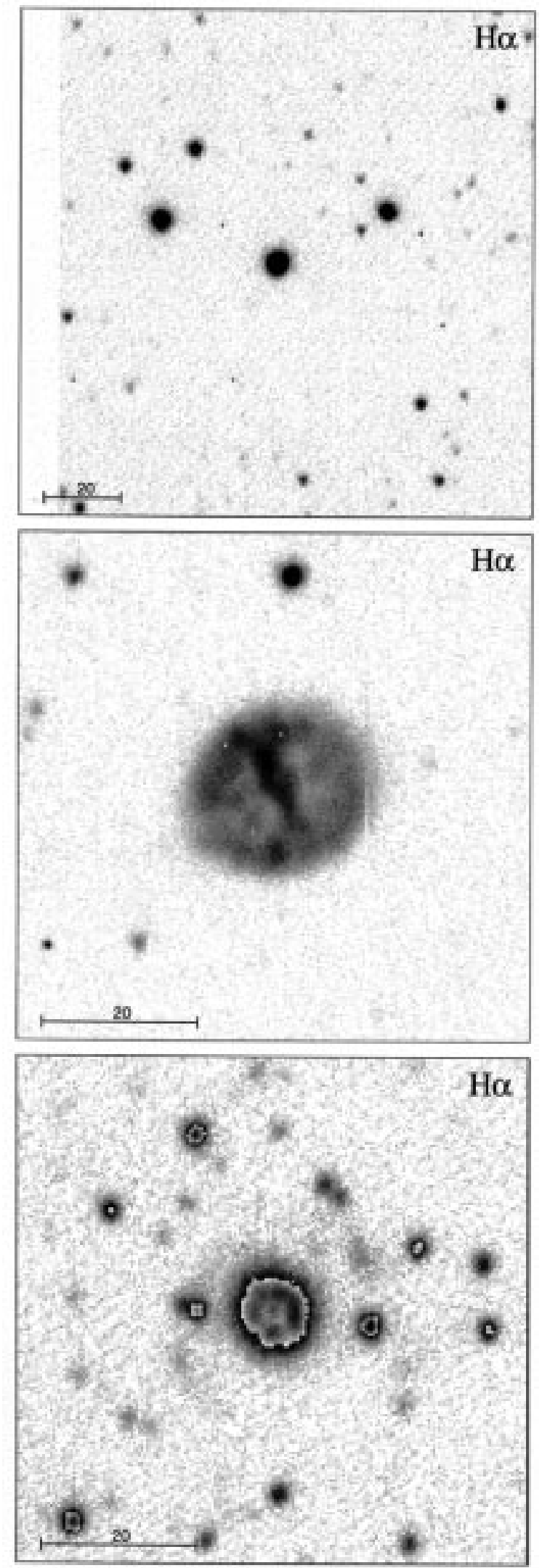

Fig. 15. He 2-35 (top), He 2-37 (middle), and He 2-39 (bottom)
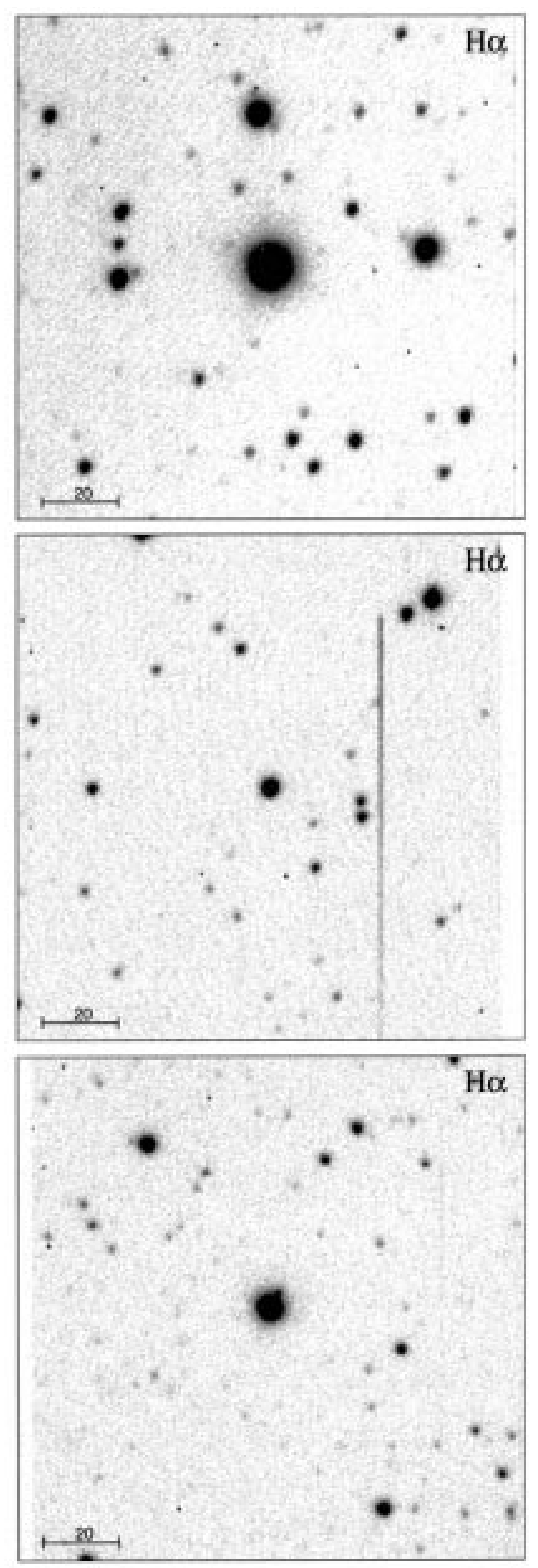

Fig. 16. He 2-47 (top), He 2-63 (middle), and He 2-68 (bottom) 

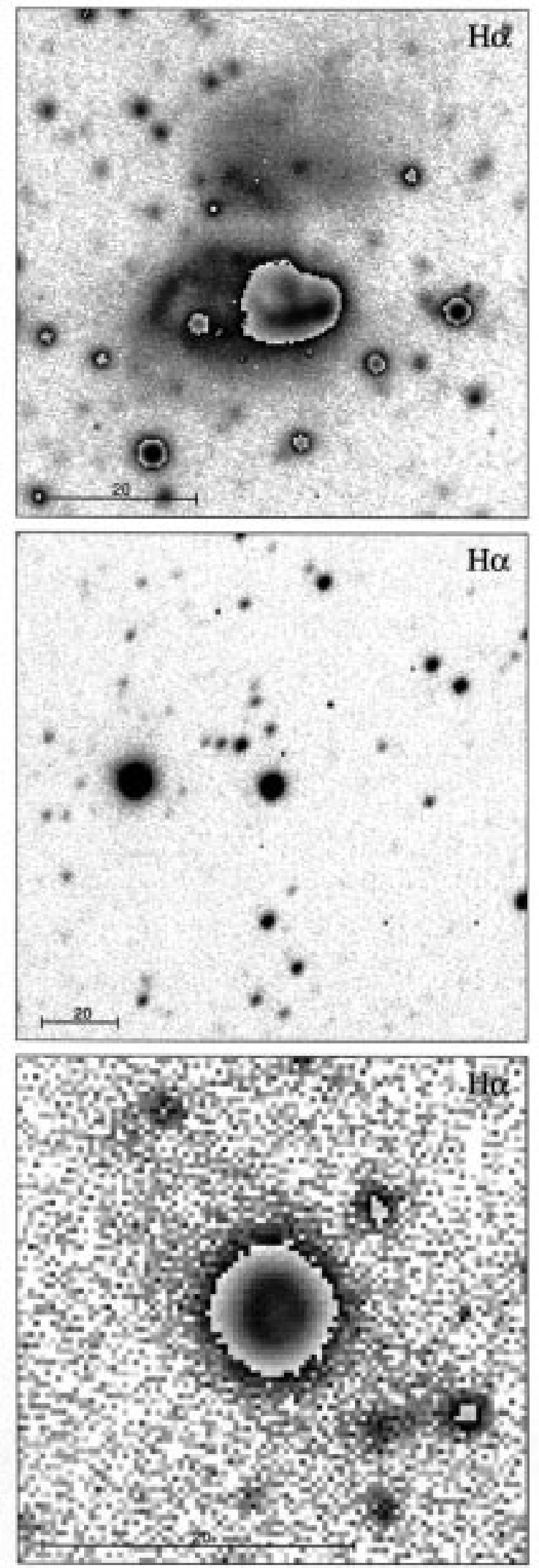

Fig. 17. He 2-77 (top), He 2-78 (middle), and He 2-81 (bottom)
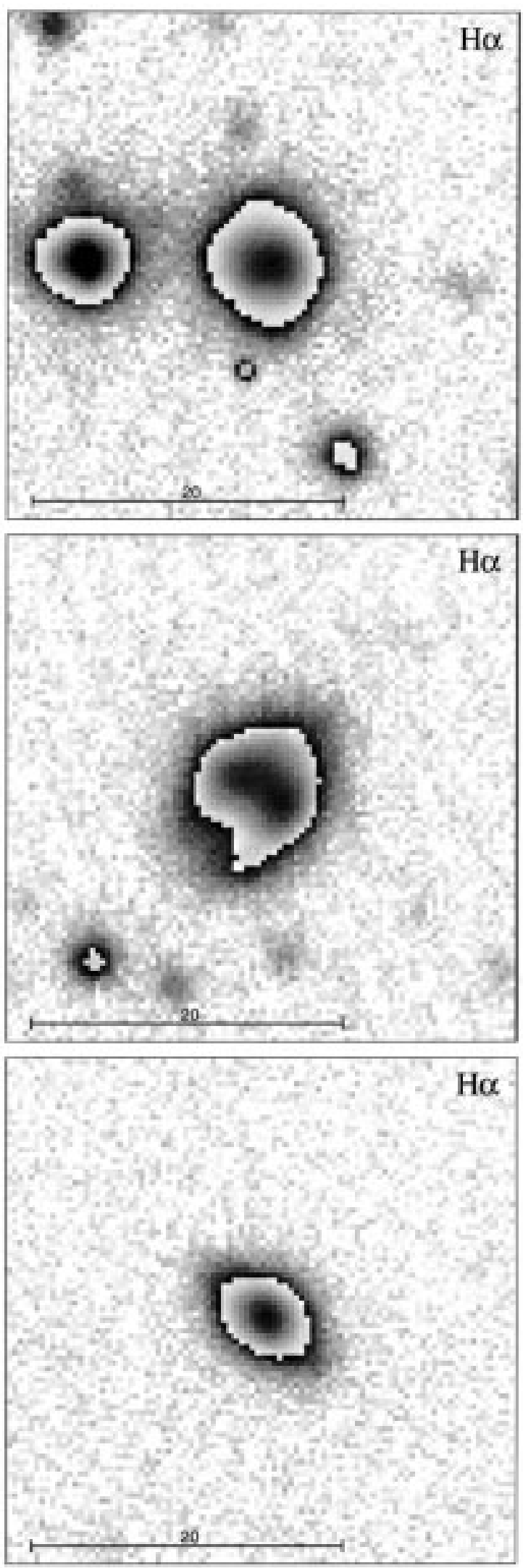

Fig. 18. He 2-83 (top), He 2-85 (middle), and He 2-86 (bottom) 

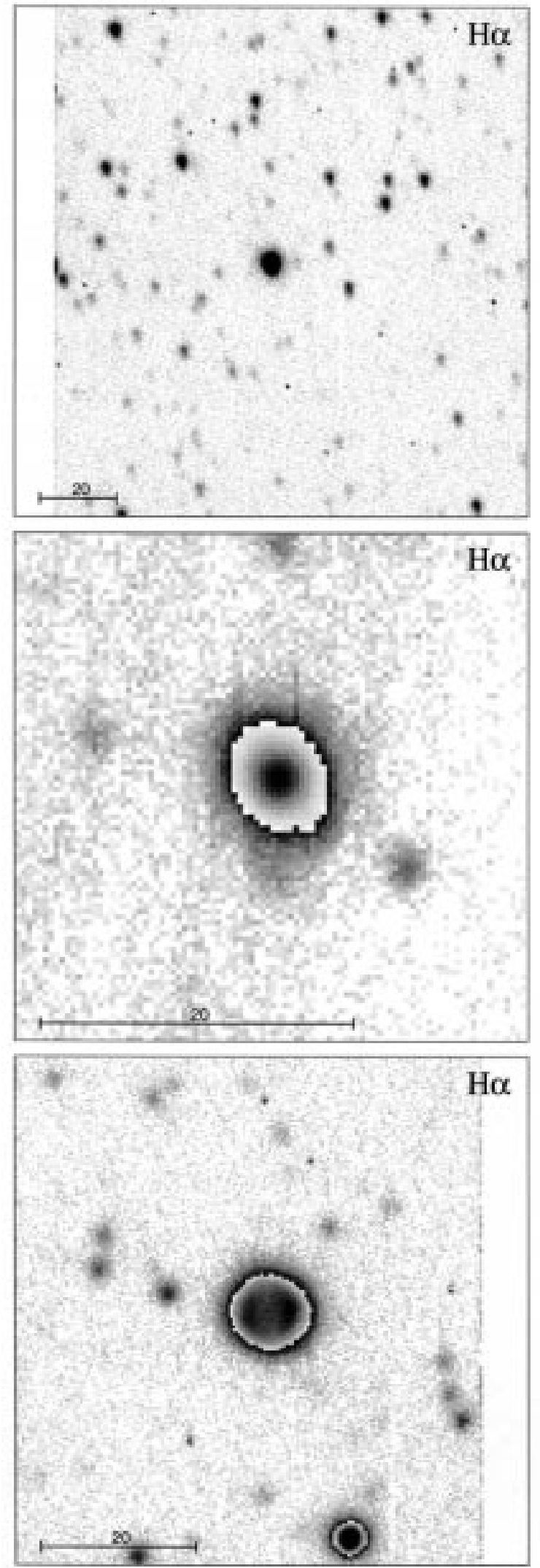

Fig. 19. He 2-88 (top), He 2-96 (middle), and He 2-102 (bottom)
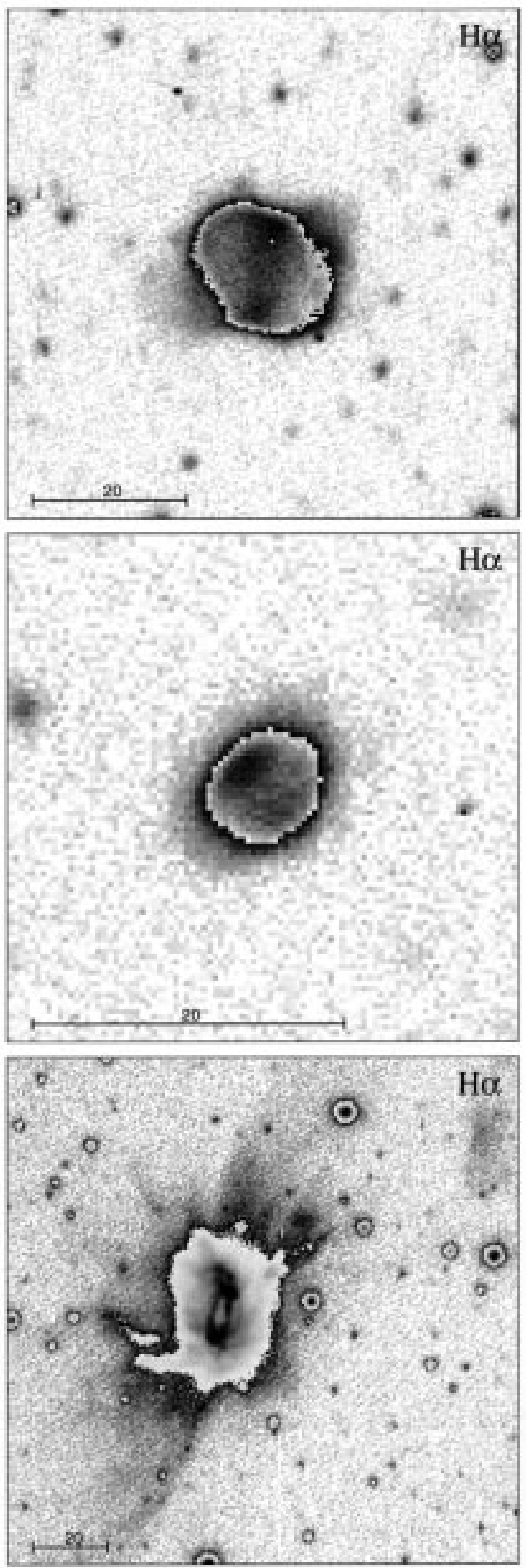

Fig. 20. He 2-103 (top), He 2-109 (middle), and He 2-111 (bottom) 

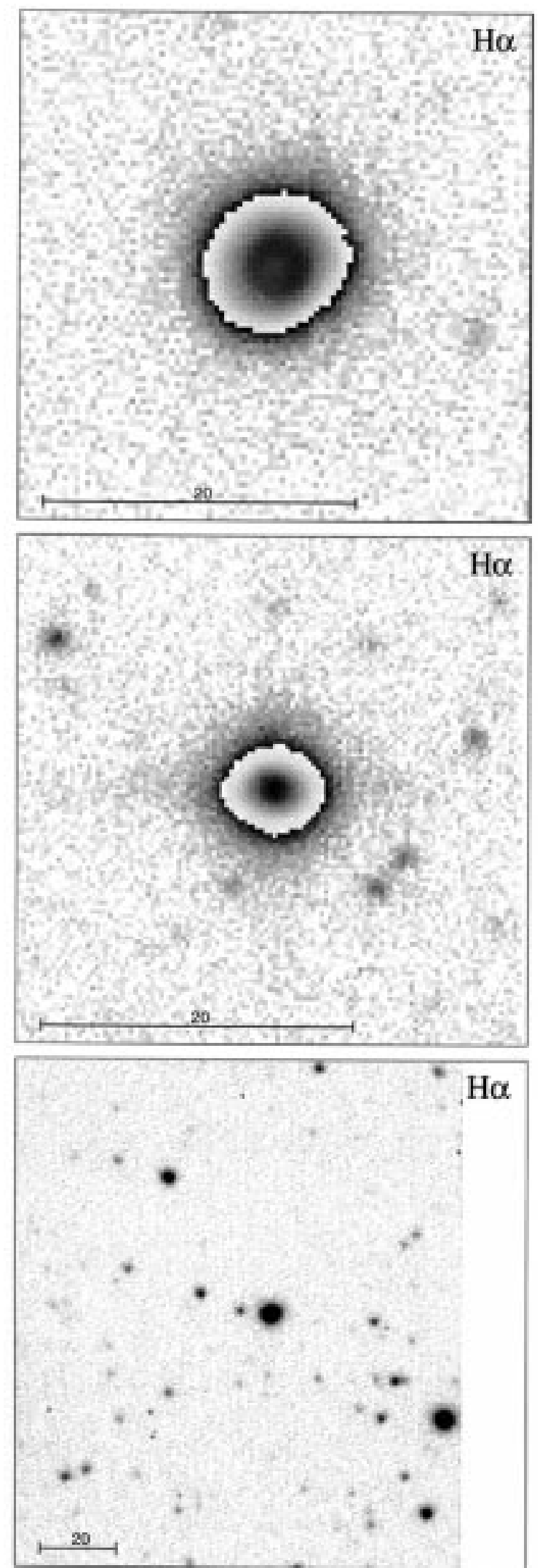

Fig. 21. He 2-112 (top), He 2-115 (middle), and He 2-129 (bottom)
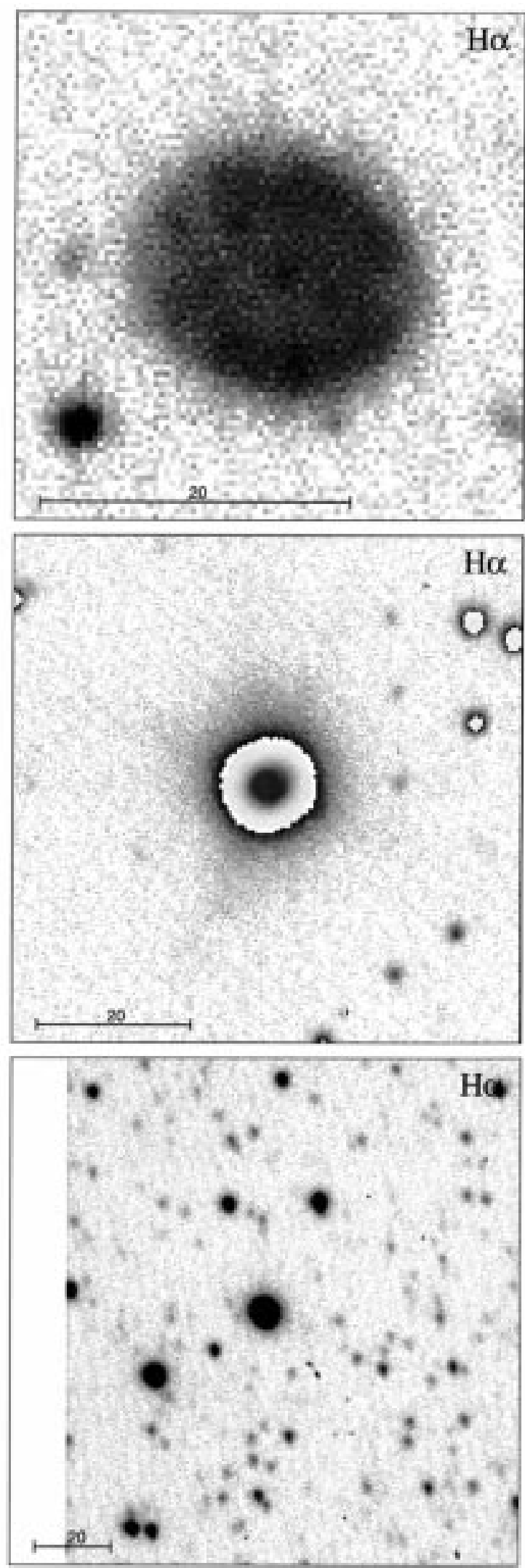

Fig. 22. He 2-132 (top), He 2-138 (middle), and He 2-140 (bottom) 

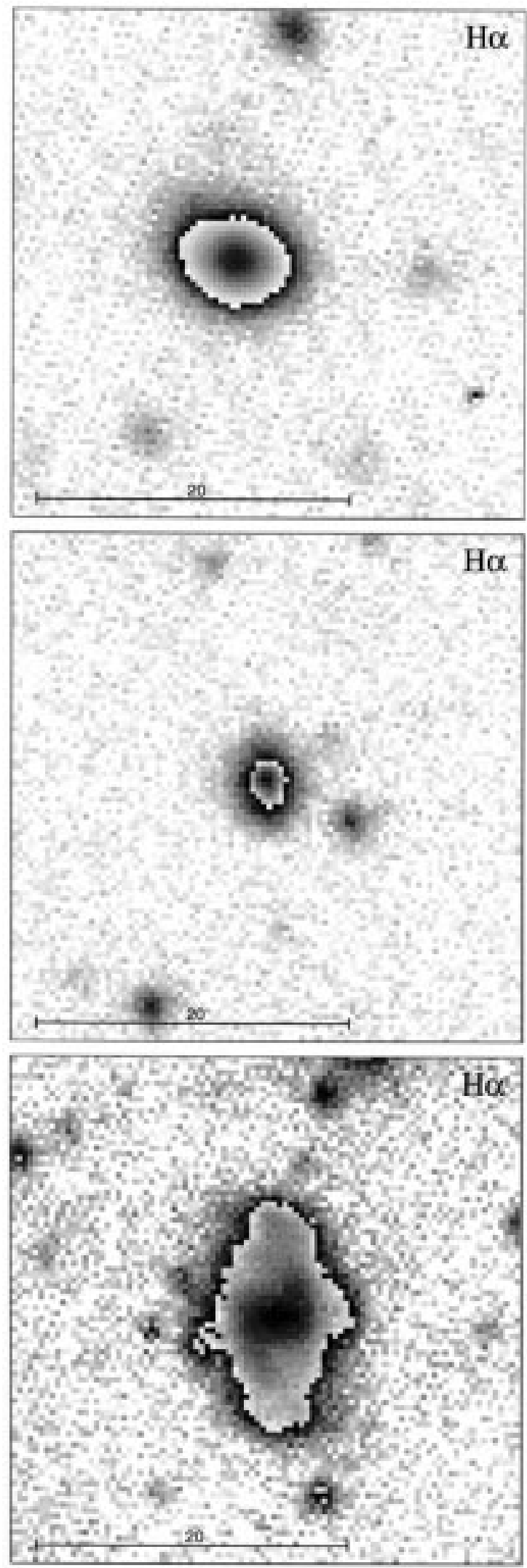

Fig. 23. He 2-143 (top), He 2-147 (middle), and He 2-159 (bottom)
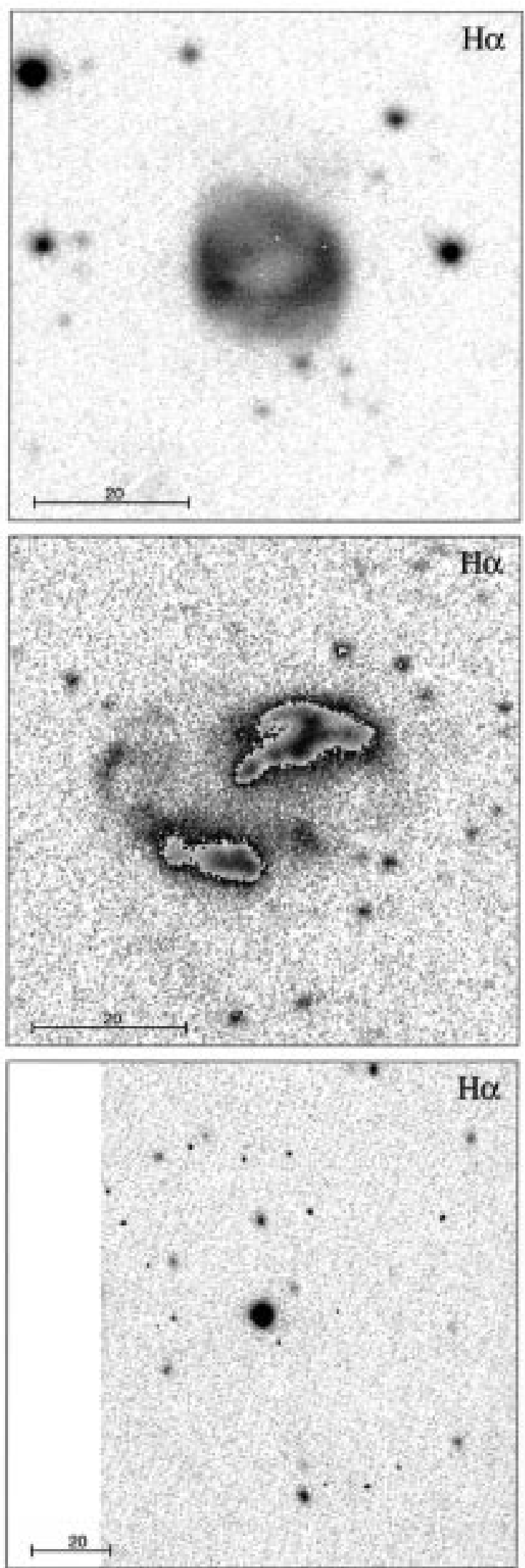

Fig. 24. He 2-163 (top), He 2-207 (middle), and He 2-436 (bottom) 

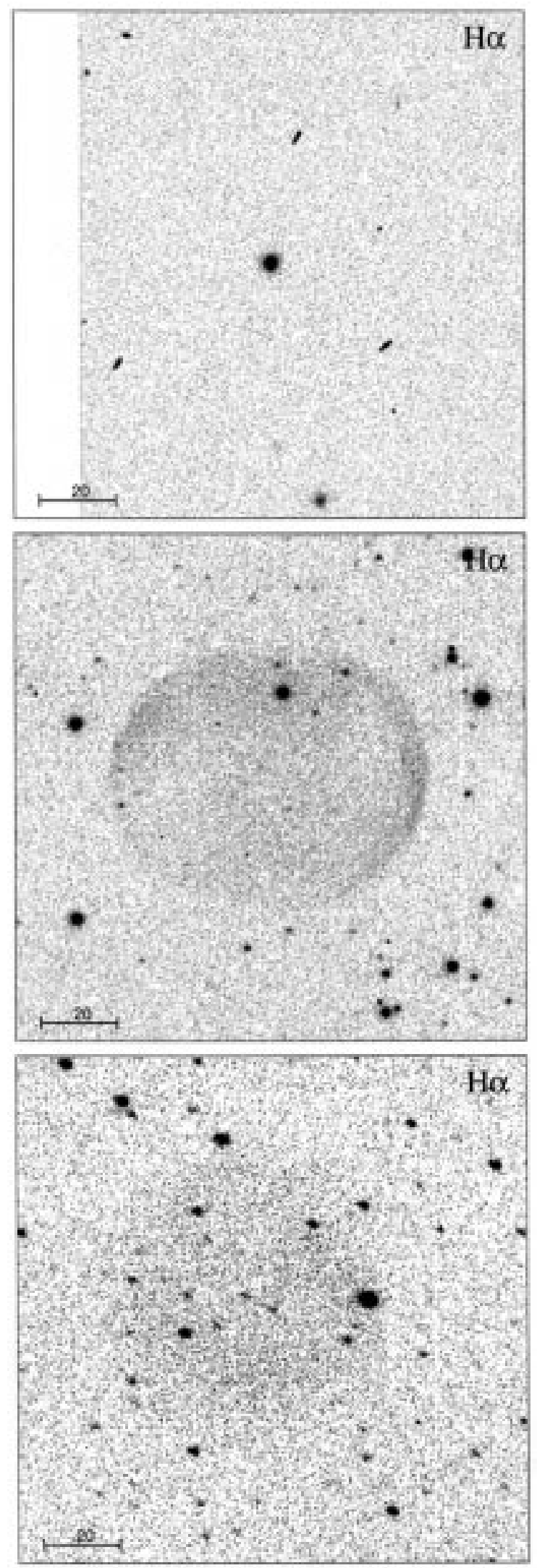

Fig. 25. K 2-7 (top), Lo 12 (middle), and Lo 13 (bottom)
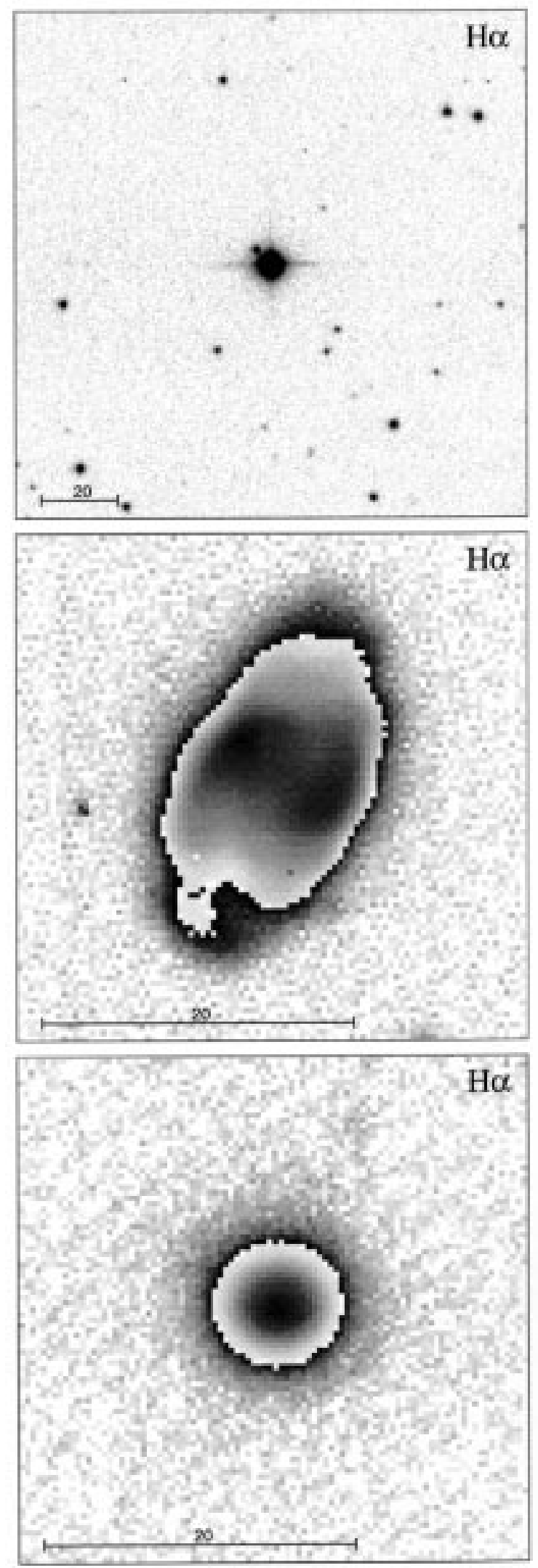

Fig. 26. Lo 14 (top), M 1-13 (middle), and M 1-14 (bottom) 

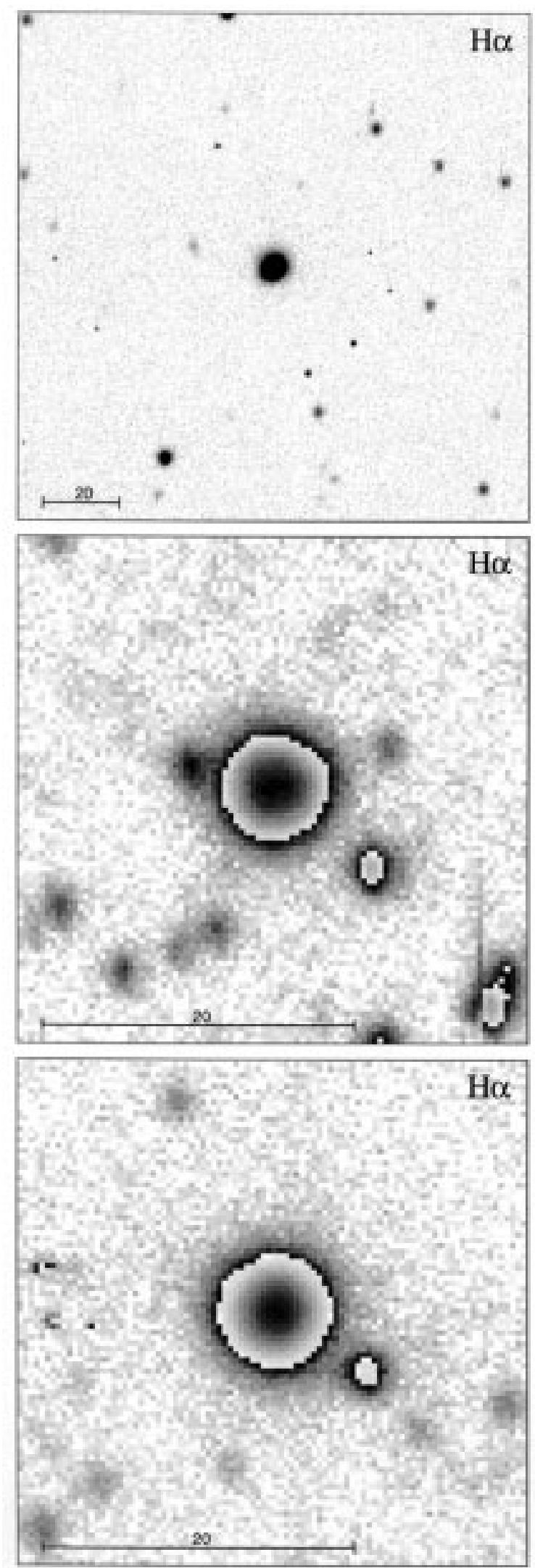

Fig. 27. M 1-17 (top), M 1-47 (middle), and M 1-62 (bottom)
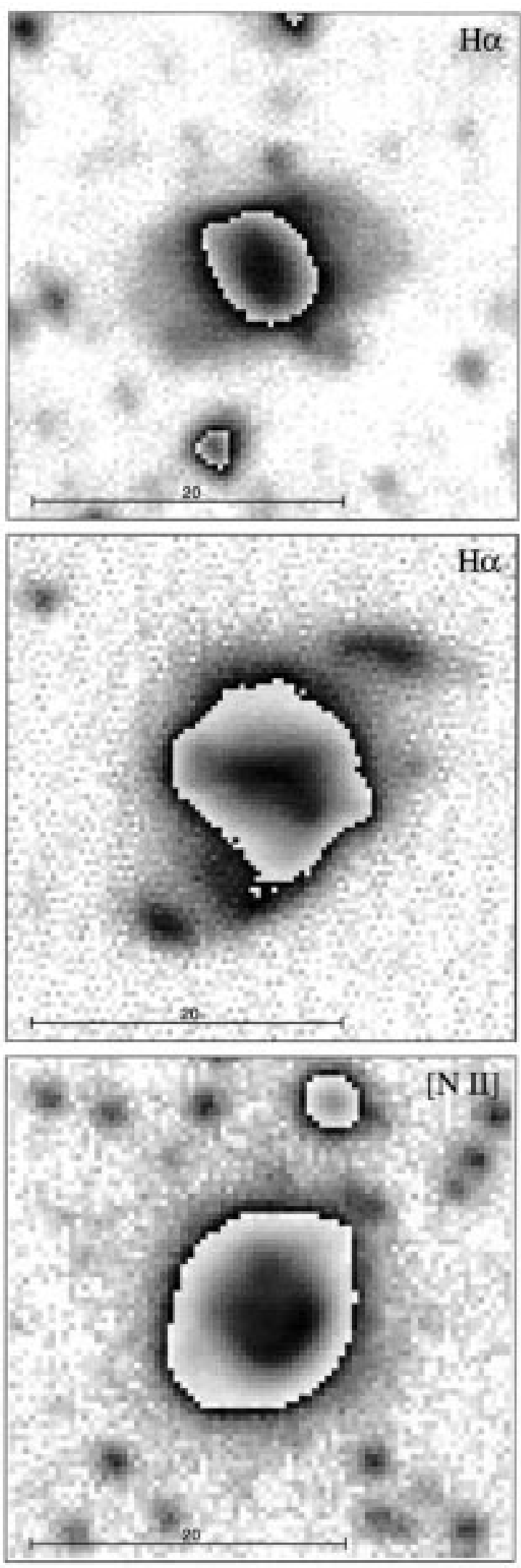

Fig. 28. Me 2-19 (top), M 3-1 (middle), and M 3-29 (bottom) 

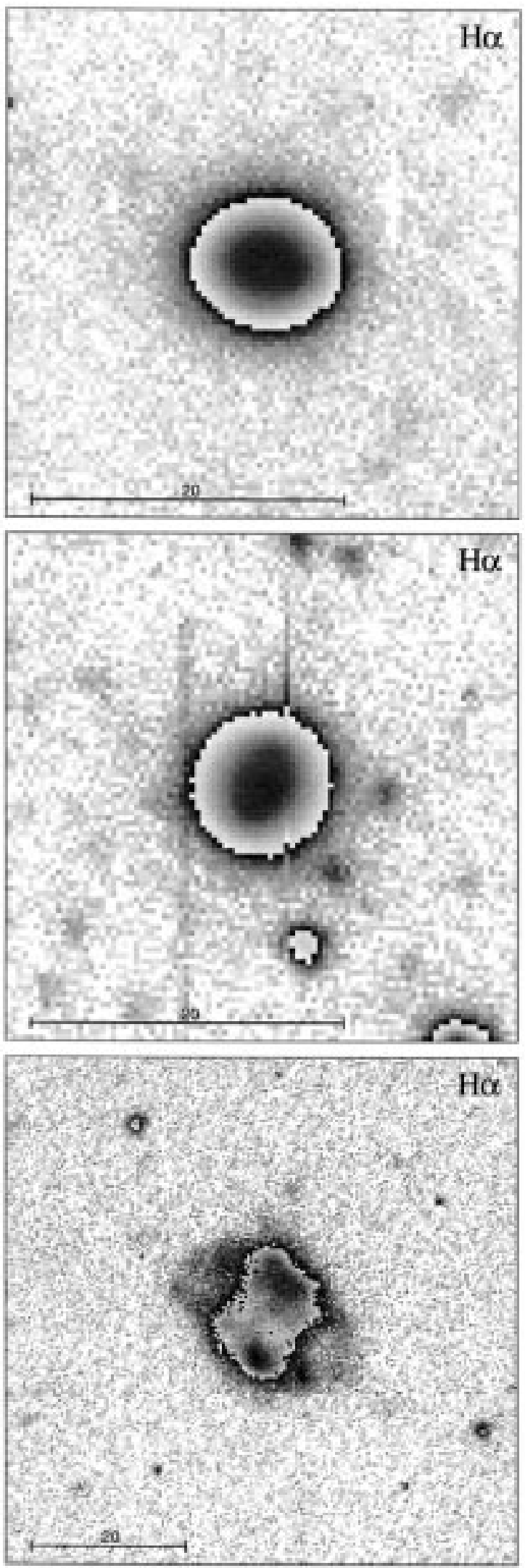

Fig. 29. M 3-32 (top), M 3-33 (middle), and M 3-39 (bottom)
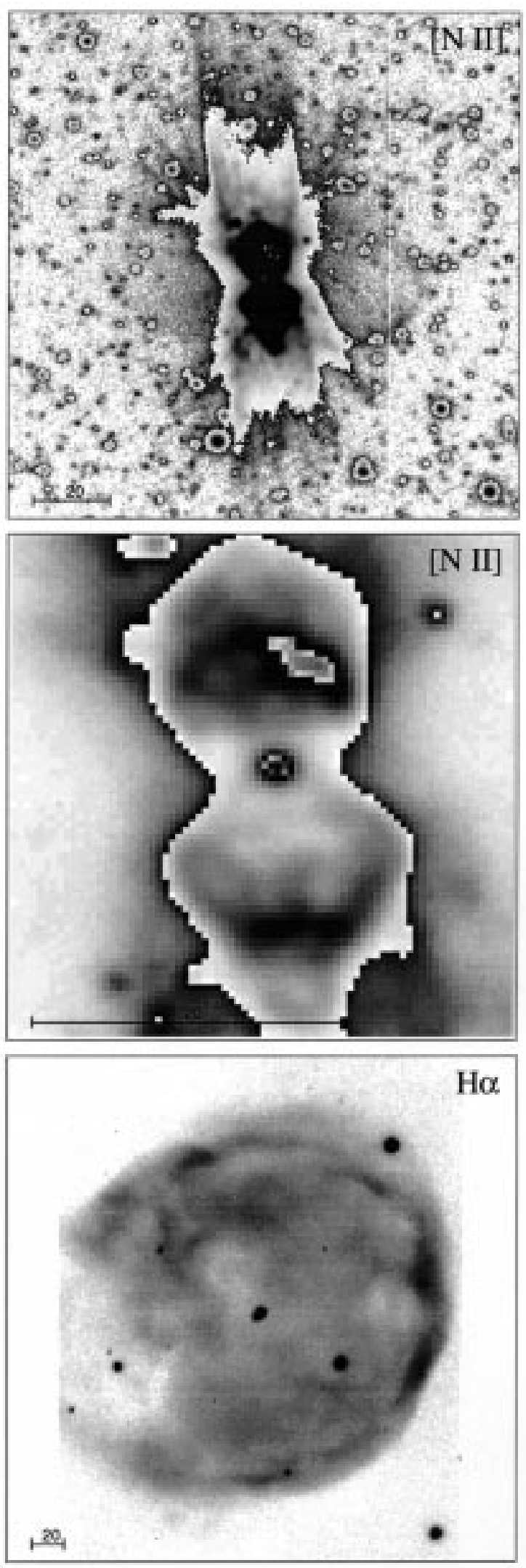

Fig. 30. Mz 3 (top \& middle), and NGC 246 (bottom) 

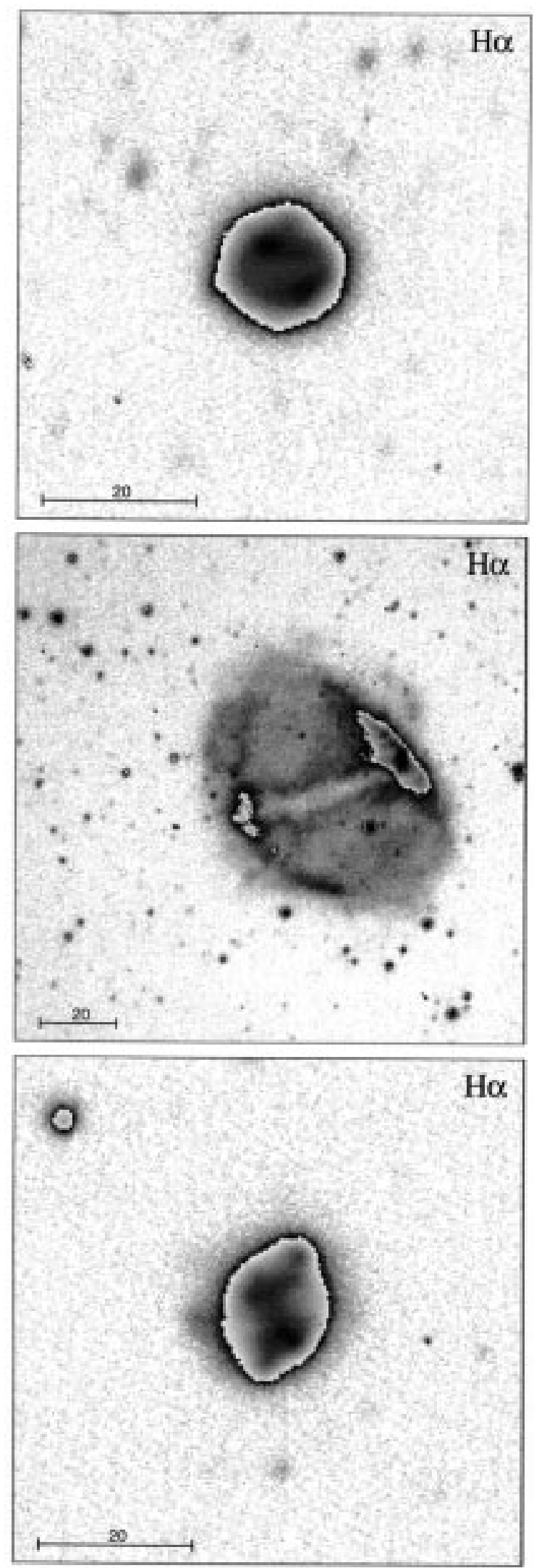

Fig. 31. NGC 3211 (top), NGC 4071 (middle), and NGC 5307 (bottom)
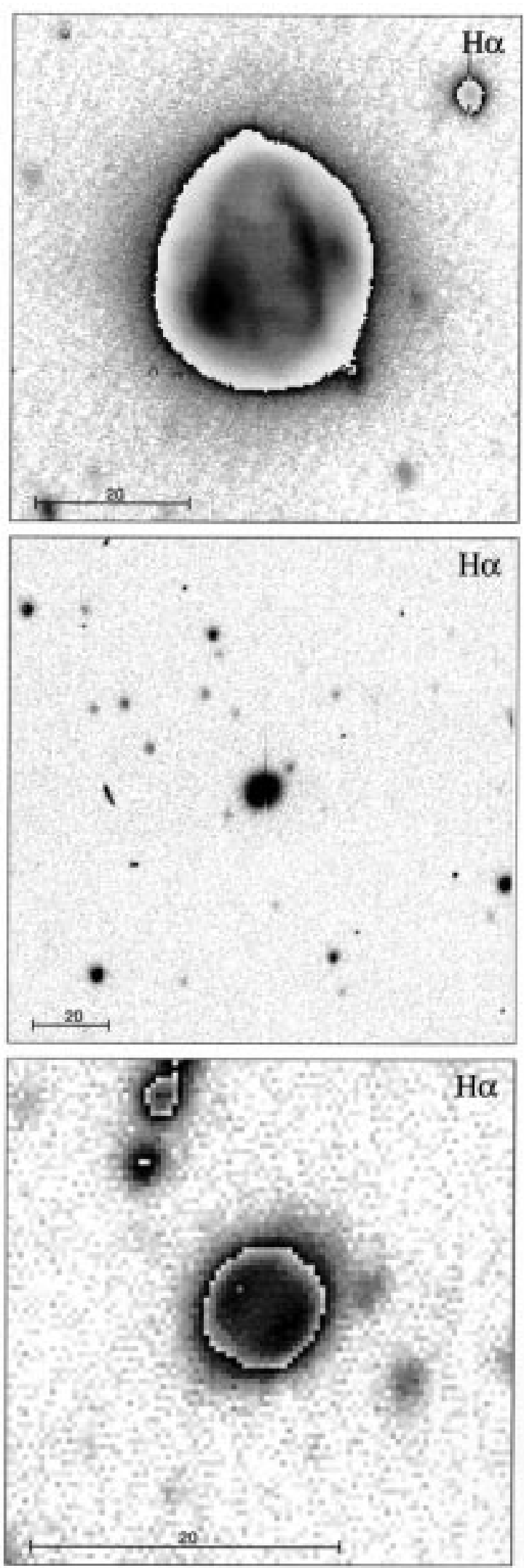

Fig. 32. NGC 6153 (top), PB 1 (middle), and Pe 1-3 (bottom) 

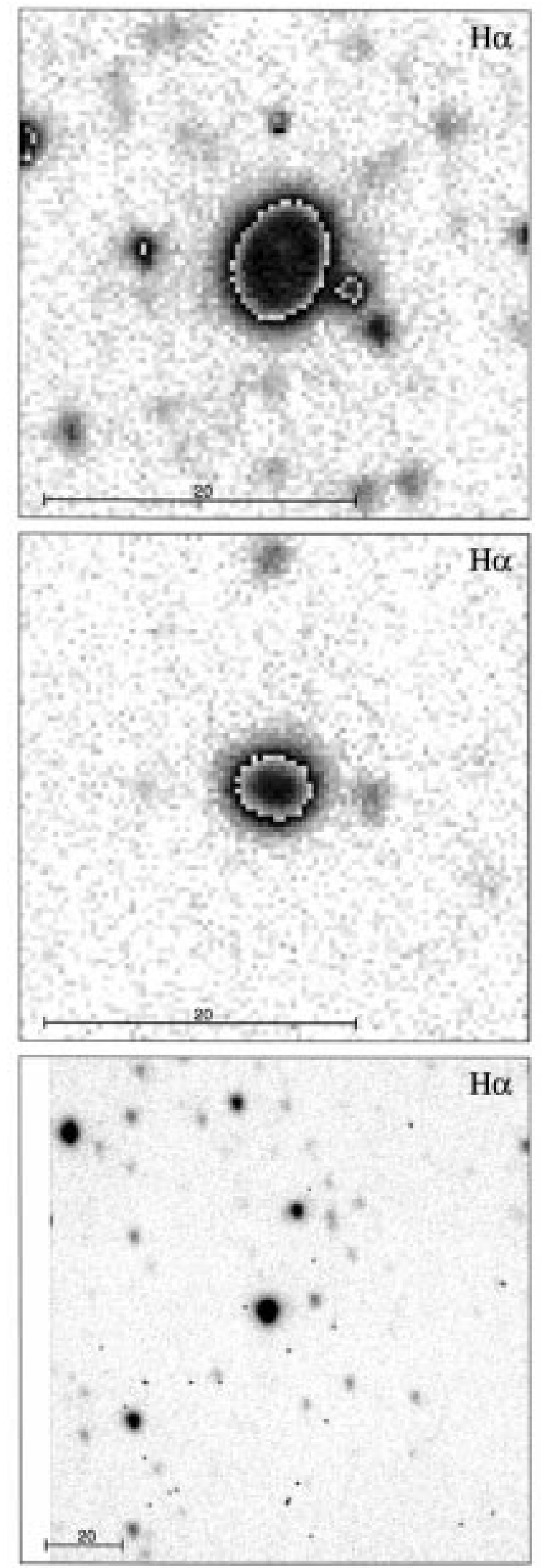

Fig. 33. Pe 1-6 (top), Pe 2-7 (middle), and Pe 2-8 (bottom)
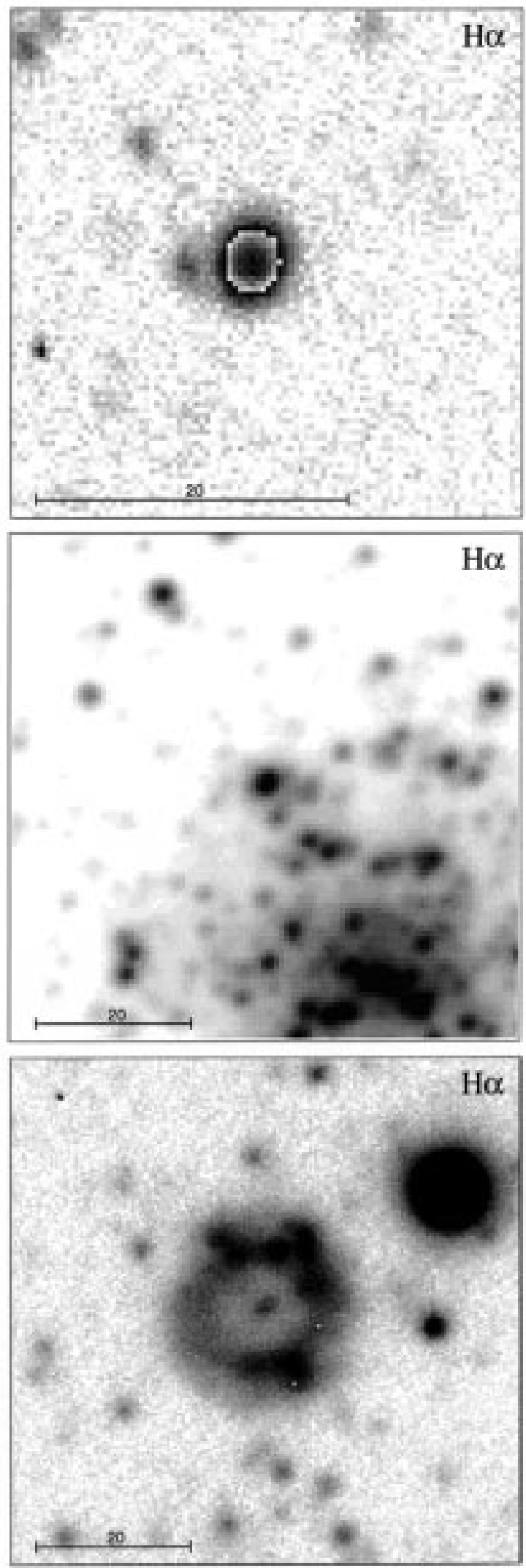

Fig. 34. Pe 2-14 (top), Ps 1 (middle), and TH 2-A (bottom) 
Table 2. List of observed objects. For each image PN size (in arcsec), exposure time (in minutes), and seeing (in arcsec) are given. Images not taken with the Dutch Telescope are indicated with the superscripted letter b or c (see Table 1), and comments are indicated by numerical superscripts. All figures have north up, east to the left

\begin{tabular}{|c|c|c|c|c|c|c|c|c|c|c|c|c|c|c|c|c|c|}
\hline \multirow[t]{2}{*}{ No. } & \multirow{2}{*}{\multicolumn{2}{|c|}{ R.A. (2000.0) Dec. }} & \multirow[t]{2}{*}{ PN G Name } & Fig. & imag & $\mathrm{H} \alpha$ & & & age $\mathrm{H}$ & & image & $\mathrm{OII}$ & & imag & ge $[N$ & N II] & \\
\hline & & & & & size & exp. & seeing & $g$ size & exp. & seeing & size & exp. & seeing & size & exp. & seein & \\
\hline 1 & 004703.8 & -115222 & $118.8-74.7$ NGC 246 & 30 & $>272 \times 239$ & 5 & 2.2 & & & & & & & & & & \\
\hline 2 & 050308.0 & -153613 & $215.5-30.8 \mathrm{~A} 7$ & 12 & stellar & 15 & 1.6 & & & & & & & & & & \\
\hline 3 & $07 \quad 0246.7$ & -134250 & $226.4-03.7 \mathrm{~PB} 1$ & 32 & $11 \times 10$ & 5 & 1.9 & & & & & & & & & & \\
\hline 4 & $07 \quad 0249.7$ & -313541 & $242.6-11.6 \mathrm{M} \mathrm{3-} 1$ & 28 & $28 \times 18$ & 5 & 1.6 & & & & & & & & & & \\
\hline 5 & 071449.5 & -275018 & $240.3-07.6 \mathrm{M} \mathrm{3-} 2$ & 1 & $35 \times 16$ & 1 & 1.7 & & & & $9 \times 6$ & 5 & 1.7 & $34 \times 16$ & 5 & $51.5^{c}$ & $c$ \\
\hline 6 & $0721 \quad 14.9$ & $\begin{array}{lll}-18 & 08 & 36\end{array}$ & $232.4-01.8 \mathrm{M} 1-13$ & 26 & $26 \times 15$ & 5 & 1.4 & & & & & & & & & & \\
\hline 7 & 072756.5 & -201323 & $234.9-01.4 \mathrm{M} \quad 1-14$ & 26 & 14 & 5 & 1.8 & & & & & & & & & & \\
\hline 8 & $07 \quad 40 \quad 22.2$ & -113230 & $228.8+05.3 \mathrm{M} 1-17$ & 27 & $9 \times 8$ & 5 & 1.9 & & & & & & & & & & \\
\hline 9 & $\begin{array}{lll}08 & 09 & 01.3\end{array}$ & -324015 & $250.3+00.1$ A 26 & 12 & $37 \times 32$ & 5 & 1.3 & & & & & & & & & & \\
\hline 10 & 083708.0 & -392624 & $259.1+00.9$ Не $2-11$ & 13 & $123 \times 64$ & 5 & 1.5 & & & & & & & & & & \\
\hline 11 & $0908 \quad 40.8$ & -531908 & $273.2-03.7$ He $2-18$ & 14 & $19 \times 14$ & 5 & 1.8 & & & & & & & & & & \\
\hline 12 & 091600.5 & -363732 & $261.9+08.5$ NGC 2818 & 10 & $123 \times 55$ & 15 & 1.2 & & & & $79 \times 49$ & 20 & 1.4 & & & & \\
\hline 13 & 092206.4 & -540947 & $275.2-02.9$ He $2-28$ & 14 & 12 & 5 & 1.9 & & & & & & & & & & \\
\hline 14 & 092444.8 & -543620 & $275.8-02.9$ Не $2-29$ & 14 & $20 \times 16$ & 5 & 1.9 & & & & & & & & & & \\
\hline 15 & 092703.5 & -560618 & 277.1-03.8 NGC 2899 & 11 & $140 \times 69$ & 20 & 1.5 & & & & $82 \times 57$ & 30 & 1.5 & & & & \\
\hline 16 & 094136.4 & -495745 & $274.6+02.1$ He $2-35$ & 15 & 8 & 5 & 1.7 & & & & & & & & & & \\
\hline 17 & $0947 \quad 24.3$ & $-48 \quad 5819$ & $274.6+03.5$ He $2-37$ & 15 & $27 \times 24$ & 5 & 1.5 & & & & & & & & & & \\
\hline 18 & 100350.0 & -604348 & $283.8-04.2$ Не $2-39$ & 15 & $14 \times 13$ & 5 & 1.8 & & & & & & & & & & \\
\hline 19 & 100922.0 & -805130 & 296.6-20.0 NGC 3195 & 2 & $43 \times 35$ & 15 & 1.5 & $33 \times 28$ & 30 & 1.6 & $44 \times 33$ & 20 & 2.0 & & & & \\
\hline 20 & 101750.4 & -624009 & $286.3-04.8$ NGC 3211 & 31 & 21 & 5 & 2.3 & & & & & & & & & & \\
\hline 21 & 102309.1 & -603235 & $285.6-02.7 \mathrm{He} 2-47$ & 16 & 18 & 5 & 2.1 & & & & & & & & & & \\
\hline 22 & 104120.0 & -560918 & $285.4+02.2$ Pe $2-7$ & 33 & 8 & 5 & 1.9 & & & & & & & & & & \\
\hline 23 & 104431.5 & -613941 & 288.4-02.4Pe 1- 3 & 32 & $14 \times 10$ & 5 & 1.9 & & & & & & & & & & \\
\hline 24 & 112400.4 & -525121 & $289.8+07.7$ He $2-63$ & 16 & 6 & 5 & 1.8 & & & & & & & & & & \\
\hline 25 & 112759.2 & -595732 & $292.6+01.2$ NGC 3699 & 11 & $88 \times 59$ & 15 & 1.2 & & & & $>114 \times 94$ & 15 & 1.1 & & & & \\
\hline 26 & 112848.0 & -600633 & $292.8+01.1$ He $2-67$ & 2 & $16 \times 10$ & 5 & 1.8 & 5 & 5 & 1.7 & $8 \times 7$ & 5 & 1.9 & & & & \\
\hline 27 & 113146.5 & -655814 & $294.9-04.3$ Не $2-68$ & 16 & 9 & 5 & 1.6 & & & & & & & & & & \\
\hline $28^{1}$ & 113513.0 & -601654 & $293.6+01.2$ He $2-70$ & 3 & $89 \times 55$ & 15 & 1.2 & $91 \times 66$ & 30 & 1.3 & & & & & & & \\
\hline 29 & 120415.3 & $-67 \quad 1835$ & 298.3-04.8 NGC 4071 & 31 & $86 \times 67$ & 15 & 1.2 & & & & & & & & & & \\
\hline 30 & 120826.0 & -641212 & $298.2-01.7 \mathrm{He} 2-76$ & 3 & $43 \times 21$ & 5 & 1.7 & & & & $20 \times 18$ & 10 & 1.7 & & & & \\
\hline 31 & 120901.0 & -630706 & $298.1-00.7$ He $2-77$ & 17 & $42 \times 24$ & 20 & 1.8 & & & & & & & & & & \\
\hline 32 & 120911.0 & -584236 & $297.4+03.7$ He $2-78$ & 17 & 9 & 5 & 2.1 & & & & & & & & & & \\
\hline 33 & 122303.0 & -640206 & $299.8-01.3$ Не $2-81$ & 17 & 11 & 10 & 1.7 & & & & & & & & & & \\
\hline 34 & 122353.0 & $\begin{array}{llll}-60 & 13 & 12\end{array}$ & $299.5+02.4$ He $2-82$ & 4 & $31 \times 25$ & 20 & 1.8 & & & & $24 \times 21$ & 20 & 1.9 & & & & \\
\hline 35 & 122846.0 & -620536 & $300.2+00.6$ He $2-83$ & 18 & 11 & 10 & 1.9 & & & & & & & & & & \\
\hline 36 & 122847.0 & -634436 & $300.4-00.9$ He $2-84$ & 4 & $38 \times 30$ & 4 & $1.1^{b}$ & & & & $17 \times 9$ & 4 & $1.1^{b}$ & & & & \\
\hline 37 & 123008.0 & -635300 & $300.5-01.1$ He $2-85$ & 18 & $16 \times 12$ & 5 & 2.0 & & & & & & & & & & \\
\hline 38 & 123030.4 & -645110 & $300.7-02.0$ He $2-86$ & 18 & $12 \times 8$ & 10 & 1.8 & & & & & & & & & & \\
\hline 39 & 130548.0 & -573924 & $304.8+05.1$ He $2-88$ & 19 & stellar & 10 & 1.8 & & & & & & & & & & \\
\hline 40 & 132234.8 & -632056 & $306.4-00.6 \mathrm{Th} 2-\mathrm{A}$ & 34 & $30 \times 28$ & 5 & 2.4 & & & & & & & & & & \\
\hline 41 & 133342.0 & -655830 & 307.2-03.4NGC 5189 & 11 & $185 \times 152$ & 4 & $1.6^{b}$ & & & & $179 \times 131$ & 4 & $1.4^{b}$ & & & & \\
\hline 42 & 134236.0 & -612230 & $309.0+00.8$ Не $2-96$ & 19 & $11 \times 8$ & 5 & 1.9 & & & & & & & & & & \\
\hline 43 & 135103.4 & -511216 & $312.3+10.5$ NGC 5307 & 31 & $25 \times 20$ & 5 & 2.2 & & & & & & & & & & \\
\hline 44 & 135813.3 & -585430 & $311.4+02.8$ He $2-102$ & 19 & 15 & 5 & 1.9 & & & & & & & & & & \\
\hline 45 & 140536.9 & -644057 & $310.7-02.9$ He $2-103$ & 20 & $31 \times 24$ & 5 & 1.5 & & & & & & & & & & \\
\hline 46 & 142049.0 & -552754 & $315.4+05.2$ He $2-109$ & 20 & $14 \times 9$ & 5 & 1.8 & & & & & & & & & & \\
\hline 47 & 143318.3 & -604945 & $315.0-00.3$ He $2-111$ & 20 & $>143 \times 76$ & 5 & 1.6 & & & & & & & & & & \\
\hline 48 & 144030.0 & -523451 & $319.2+06.8$ He $2-112$ & 21 & 13 & 5 & 1.8 & & & & & & & & & & \\
\hline 49 & 150408.8 & -605321 & $318.3-02.0$ He $2-114$ & 4 & $45 \times 22$ & 5 & 1.7 & & & & $20 \times 18$ & 5 & 1.7 & & & & \\
\hline 50 & $1505 \quad 16.7$ & $\begin{array}{lll}-55 & 11 & 12\end{array}$ & $321.3+02.8$ He $2-115$ & 21 & 12 & 4 & $1.2^{b}$ & & & & & & & & & & \\
\hline 51 & 150600.8 & $\begin{array}{lll}-612124 & 24\end{array}$ & $318.3-02.5$ He $2-116$ & 5 & $49 \times 45$ & 15 & 2.8 & & & & $47 \times 42$ & 15 & 2.8 & & & & \\
\hline 52 & 151039.9 & -644019 & $317.1-05.7$ He $2-119$ & 5 & $113 \times 70$ & 20 & 2.2 & & & & $70 \times 66$ & 15 & 2.6 & & & & \\
\hline 53 & 151156.1 & -553951 & $321.8+01.9$ He $2-120$ & 5 & $48 \times 31$ & 20 & 1.7 & & & & $28 \times 22$ & 5 & 1.7 & & & & \\
\hline 54 & 152342.2 & -570918 & $322.4-00.1$ Pe $2-8$ & 33 & 8 & 15 & 2.1 & & & & & & & & & & \\
\hline 55 & 152532.6 & -525043 & $325.0+03.2$ Не $2-129$ & 21 & 8 & 5 & 1.7 & & & & & & & & & & \\
\hline 56 & 153800.5 & -584451 & $323.1-02.5$ He $2-132$ & 22 & $21 \times 18$ & 5 & 2.0 & & & & & & & & & & \\
\hline 57 & 155116.3 & -484501 & $330.7+04.1$ Cn $1-1$ & 12 & stellar & 5 & 1.8 & & & & & & & & & & \\
\hline 58 & 155601.3 & $\begin{array}{lll}-66 & 09 & 11\end{array}$ & $320.1-09.6$ He $2-138$ & 22 & 22 & 5 & 1.6 & & & & & & & & & & \\
\hline 59 & 155809.0 & -554153 & $327.1-01.8$ He $2-140$ & 22 & 12 & 10 & 2.1 & & & & & & & & & & \\
\hline 60 & 160059.6 & -550539 & $327.8-01.6$ He $2-143$ & 23 & $11 \times 9$ & 10 & 2.4 & & & & & & & & & & \\
\hline 61 & 160826.3 & $-3708 \quad 48$ & $340.8+10.8$ Lo 12 & 25 & $83 \times 70$ & 4 & $1.3^{b}$ & & & & & & & & & & \\
\hline 62 & 160859.4 & $\begin{array}{lll}-51 & 02 & 02\end{array}$ & $331.4+00.5$ He $2-145$ & 6 & $32 \times 17$ & 20 & 1.4 & & & & $11 \times 10$ & 20 & 1.4 & & & & \\
\hline 63 & 160944.0 & -305400 & $345.5+15.1$ Lo 13 & 25 & 66 & 4 & $1.4^{b}$ & & & & & & & & & & \\
\hline 64 & 161041.0 & -545732 & $328.9-02.4$ He $2-146$ & 6 & $56 \times 35$ & 20 & 1.2 & & & & $23 \times 19$ & 30 & 1.3 & & & & \\
\hline $65^{2}$ & 161142 & -5118 & PK $331+0^{\circ} 2$ Lo 14 & 26 & stellar & 1 & $1.1 b$ & & & & & & & & & & \\
\hline 66 & 161401.0 & -565930 & $327.9-04.3$ He $2-147$ & 23 & $7 \times 5$ & 20 & 1.6 & & & & & & & & & & \\
\hline 67 & 161432.1 & -545704 & $329.3-02.8 \mathrm{Mz} 2$ & 10 & & & & $23 \times 21$ & 30 & 1.5 & $44 \times 29$ & 20 & 1.4 & & & & \\
\hline 68 & 161520.3 & -491325 & $333.4+01.1$ He $2-152$ & 6 & $28 \times 22$ & 15 & 1.6 & & & & $11 \times 10$ & 15 & 1.9 & & & & \\
\hline 69 & 161713.6 & -515906 & $331.7-01.0 \mathrm{Mz} 3$ & 30 & & & & & & & & & & $133 \times 84$ & 3 & $31.4^{c}$ & \\
\hline 70 & $1617 \quad 14.0$ & -533203 & $330.6-02.1$ He $2-153$ & 7 & $29 \times 16$ & 15 & 1.5 & & & & $15 \times 12$ & 15 & 1.5 & & & & \\
\hline 71 & 161923.1 & -421536 & $338.8+05.6$ He $2-155$ & 7 & $48 \times 21$ & 30 & 1.3 & & & & $49 \times 20$ & 40 & 1.5 & & & & \\
\hline 72 & 162354.7 & $-4642 \quad 12$ & $336.2+01.9$ Pe $1-6$ & 33 & $11 \times 9$ & 15 & 1.4 & & & & & & & & & & \\
\hline
\end{tabular}


Table 2. continued

\begin{tabular}{|c|c|c|c|c|c|c|c|c|c|c|c|c|c|c|c|}
\hline \multirow[t]{2}{*}{ No. } & \multirow{2}{*}{\multicolumn{2}{|c|}{ R.A. (2000.0) Dec. }} & \multirow[t]{2}{*}{ PN G Name } & \multirow[t]{2}{*}{ Fig. } & \multicolumn{3}{|c|}{ image $\mathrm{H} \alpha$} & image $\mathrm{H} \beta$ & \multicolumn{3}{|c|}{ image [O III $]$} & \multicolumn{4}{|c|}{ image $[\mathrm{N} \mathrm{II}]$} \\
\hline & & & & & size & exp. & & igsize exp. seeing & size & exp. & seeing & size & exp. & seein & \\
\hline 73 & 162421.1 & -543603 & $330.6-03.6$ He $2-159$ & 23 & $17 \times 13$ & 15 & 1.5 & & & & & & & & \\
\hline 74 & 162437.2 & -532234 & $331.5-02.7$ He $2-161$ & 7 & $24 \times 15$ & 15 & 1.5 & & $17 \times 12$ & 15 & 1.3 & & & & \\
\hline 75 & 162930.4 & $\begin{array}{lll}-59 & 09 & 22\end{array}$ & $327.8-07.2$ He $2-163$ & 24 & $26 \times 20$ & 5 & 1.7 & & & & & & & & \\
\hline 76 & 162953.2 & $\begin{array}{lll}-532304 & 04\end{array}$ & $332.0-03.3$ Не $2-164$ & 8 & 18 & 15 & 1.5 & & 18 & 15 & 1.4 & & & & \\
\hline 77 & 162959.6 & -540937 & $331.5-03.9$ Не $2-165$ & 8 & $71 \times 64$ & 15 & 1.4 & & $53 \times 41$ & 15 & 1.6 & & & & \\
\hline 78 & 163130.9 & $\begin{array}{lll}-40 & 15 & 22\end{array}$ & $341.8+05.4$ NGC 6153 & 32 & $42 \times 41$ & 15 & 1.9 & & & & & & & & \\
\hline 79 & 163414.9 & -492120 & $335.4-01.1$ He $2-169$ & 8 & $37 \times 29$ & 15 & 1.6 & & & & & $77 \times 44$ & 15 & 1.7 & $c$ \\
\hline 80 & 170623.0 & -441312 & $342.9-02.0$ Pe $1-8$ & 12 & $25 \times 23$ & 4 & 1.0 & $b$ & $25 \times 24$ & 4 & $1.1^{b}$ & & & & \\
\hline 81 & 171932.0 & -455312 & $342.9-04.9$ Не $2-207$ & 24 & $42 \times 27$ & 4 & 1.4 & $b$ & & & & & & & \\
\hline 82 & 172111.6 & -271128 & $358.5+05.4$ M 3-39 & 29 & $30 \times 21$ & 10 & 1.6 & & & & & & & & \\
\hline 83 & 174738.3 & -220620 & $006.0+03.1$ M $1-28$ & 1 & $82 \times 47$ & 15 & 1.5 & & $23 \times 21$ & 15 & 1.8 & $103 \times 53$ & 10 & 1.5 & \\
\hline 84 & $1751 \quad 12.3$ & -345526 & $355.4-04.0$ Hf $2-1$ & 9 & $20 \times 20$ & 10 & 1.6 & & & & & $22 \times 22$ & 10 & 1.5 & \\
\hline 85 & 175346.0 & -294348 & $000.2-01.9$ M 2-19 & 28 & $18 \times 12$ & 10 & 1.8 & & & & & & & & \\
\hline 86 & $1758 \quad 15.0$ & -334736 & $357.1-04.7$ H $1-43$ & 13 & stellar & 10 & 1.9 & & & & & & & & \\
\hline 87 & 180430.0 & -345800 & $356.7-06.4$ H $1-51$ & 3 & $20 \times 17$ & 20 & 1.8 & & $16 \times 13$ & 20 & 1.7 & & & & \\
\hline 88 & 180456.0 & -325406 & $358.6-05.5$ M 3-51 & 10 & $21 \times 15$ & 10 & 1.7 & & & & & $22 \times 18$ & 10 & 1.7 & $c$ \\
\hline 89 & 182911.0 & -214654 & 011.0-05.1 M 1-47 & 27 & 9 & 10 & 1.6 & & & & & & & & \\
\hline 90 & 183000.0 & -194036 & 013.0-04.3 Pe 2-14 & 34 & $7 \times 6$ & 10 & 1.5 & & & & & & & & \\
\hline 91 & 183329.0 & -110724 & 020.9-01.1 M 1-51 & 9 & $30 \times 16$ & 20 & 1.7 & & $12 \times 8$ & 20 & 2.2 & & & & \\
\hline 92 & 183925.8 & -304036 & 004.0-11.1 M 3-29 & 28 & & & & & & & & $20 \times 18$ & 10 & 1.7 & $c$ \\
\hline 93 & 184443.0 & -252136 & $009.4-09.8$ M 3-32 & 29 & 14 & 15 & 2.3 & & & & & & & & \\
\hline 94 & $1848 \quad 12.0$ & -252848 & $009.6-10.6$ M 3-33 & 29 & 13 & 15 & 1.7 & & & & & & & & \\
\hline 95 & 185026.0 & -223424 & 012.5-09.8 M 1-62 & 27 & 12 & 15 & 1.7 & & & & & & & & \\
\hline 96 & 185538.7 & -321555 & $003.9-14.9 \mathrm{Hb} 7$ & 13 & $13 \times 12$ & 5 & 1.7 & & & & & & & & \\
\hline $97^{2}$ & 192200 & -0412 & PK $32-8^{\circ} 1$ Anon. $19 \mathrm{~h} 19 \mathrm{~m}$ & 12 & $7 \times 5$ & 4 & 1.6 & $b$ & & & & & & & \\
\hline 98 & 193207.3 & -341233 & $004.8-22.7$ He $2-436$ & 24 & 8 & 10 & 1.7 & & & & & & & & \\
\hline 99 & 194030.0 & -202700 & $019.4-19.6$ K 2- 7 & 25 & stellar & 20 & 2.2 & & & & & & & & \\
\hline 100 & 212959.4 & +121026 & $065.0-27.3$ Ps 1 & 34 & $9 \times 7$ & 3 & 2.1 & $c$ & & & & & & & \\
\hline 101 & 215930.0 & $\begin{array}{llll}-39 & 23 & 00\end{array}$ & 002.7-52.4 IC 5148-50 & 9 & $155 \times 133$ & 20 & 2.3 & & $135 \times 129$ & 20 & 2.3 & & & & \\
\hline
\end{tabular}

Table 3. Alphabetical list of objects

\begin{tabular}{lrr}
\hline Name & No. & Fig. \\
\hline A 26 & 9 & 12 \\
A 7 & 2 & 12 \\
Anon. 19h19m & 97 & 12 \\
Cn 1-1 & 57 & 12 \\
H 1-43 & 86 & 13 \\
H 1-51 & 87 & 3 \\
Hb 7 & 96 & 13 \\
He 2-11 & 10 & 13 \\
He 2-18 & 11 & 14 \\
He 2-28 & 13 & 14 \\
He 2-29 & 14 & 14 \\
He 2-35 & 16 & 15 \\
He 2-37 & 17 & 15 \\
He 2-39 & 18 & 15 \\
He 2-47 & 21 & 16 \\
He 2-63 & 24 & 16 \\
He 2-67 & 26 & 2 \\
He 2-68 & 27 & 16 \\
He 2-70 & 28 & 3 \\
He 2-76 & 30 & 3 \\
He 2-77 & 31 & 17 \\
He 2-78 & 32 & 17 \\
He 2-81 & 33 & 17 \\
He 2-82 & 34 & 4 \\
He 2-83 & 35 & 18 \\
He 2-84 & 36 & 4 \\
\hline & &
\end{tabular}

\begin{tabular}{lrr}
\hline Name & No. & Fig. \\
\hline He 2-85 & 37 & 18 \\
He 2-86 & 38 & 18 \\
He 2-88 & 39 & 19 \\
He 2-96 & 42 & 19 \\
He 2-102 & 44 & 19 \\
He 2-103 & 45 & 20 \\
He 2-109 & 46 & 20 \\
He 2-111 & 47 & 20 \\
He 2-112 & 48 & 21 \\
He 2-114 & 49 & 4 \\
He 2-115 & 50 & 21 \\
He 2-116 & 51 & 5 \\
He 2-119 & 52 & 5 \\
He 2-120 & 53 & 5 \\
He 2-129 & 55 & 21 \\
He 2-132 & 56 & 22 \\
He 2-138 & 58 & 22 \\
He 2-140 & 59 & 22 \\
He 2-143 & 60 & 23 \\
He 2-145 & 62 & 6 \\
He 2-146 & 64 & 6 \\
He 2-147 & 66 & 23 \\
He 2-152 & 68 & 6 \\
He 2-153 & 70 & 7 \\
He 2-155 & 71 & 7 \\
He 2-159 & 73 & 23 \\
\hline
\end{tabular}

\begin{tabular}{lrr}
\hline Name & No. & Fig. \\
\hline He 2-161 & 74 & 7 \\
He 2-163 & 75 & 24 \\
He 2-164 & 76 & 8 \\
He 2-165 & 77 & 8 \\
He 2-169 & 79 & 8 \\
He 2-207 & 81 & 24 \\
He 2-436 & 98 & 24 \\
Hf 2-1 & 84 & 9 \\
IC 5148-50 & 101 & 9 \\
K 2-7 & 99 & 25 \\
Lo 12 & 61 & 25 \\
Lo 13 & 63 & 25 \\
Lo 14 & 65 & 26 \\
M 1-13 & 6 & 26 \\
M 1-14 & 7 & 26 \\
M 1-17 & 8 & 27 \\
M 1-28 & 83 & 1 \\
M 1-47 & 89 & 27 \\
M 1-51 & 91 & 9 \\
M 1-62 & 95 & 27 \\
M 2-19 & 85 & 28 \\
M 3- 1 & 4 & 28 \\
M 3- 2 & 5 & 1 \\
M 3-29 & 92 & 28 \\
M 3-32 & 93 & 29 \\
M 3-33 & 94 & 29 \\
\hline
\end{tabular}

\begin{tabular}{lrr}
\hline Name & No. & Fig. \\
\hline M 3-39 & 82 & 29 \\
M 3-51 & 88 & 10 \\
Mz 2 & 67 & 10 \\
Mz 3 & 69 & 30 \\
NGC 246 & 1 & 30 \\
NGC 2818 & 12 & 10 \\
NGC 2899 & 15 & 11 \\
NGC 3195 & 19 & 2 \\
NGC 3211 & 20 & 31 \\
NGC 3699 & 25 & 11 \\
NGC 4071 & 29 & 31 \\
NGC 5189 & 41 & 11 \\
NGC 5307 & 43 & 31 \\
NGC 6153 & 78 & 32 \\
PB 1 & 3 & 32 \\
Pe 1-3 & 23 & 32 \\
Pe 1-6 & 72 & 33 \\
Pe 1-8 & 80 & 12 \\
Pe 2-7 & 22 & 33 \\
Pe 2-8 & 54 & 33 \\
Pe 2-14 & 90 & 34 \\
Ps 1 & 100 & 34 \\
Th 2-A & 40 & 34 \\
\hline
\end{tabular}

